\title{
Entropy and Variational Principle for one-dimensional Lattice Systems with a general a-priori probability: positive and zero temperature
}

\author{
A. O. Lopes, J. K. Mengue, J. Mohr and R. R. Souza
}

July 17, 2018

Instituto de Matemática, UFRGS - Porto Alegre, Brasil

\begin{abstract}
We generalize several results of the classical theory of Thermodynamic Formalism by considering a compact metric space $M$ as the state space. We analyze the shift acting on $M^{\mathbb{N}}$ and consider a general a-priori probability for defining the Transfer (Ruelle) operator. We study potentials $A$ which can depend on the infinite set of coordinates in $M^{\mathbb{N}}$. We define entropy and by its very nature it is always a nonpositive number. The concepts of entropy and transfer operator are linked. If $\mathrm{M}$ is not a finite set there exist Gibbs states with arbitrary negative value of entropy. Invariant probabilities with support in a fixed point will have entropy equal to minus infinity. In the case $M=S^{1}$, and the a-priori measure is Lebesgue $d x$, the infinite product of $d x$ on $\left(S^{1}\right)^{\mathbb{N}}$ will have zero entropy.

We analyze the Pressure problem for a Hölder potential $A$ and its relation with eigenfunctions and eigenprobabilities of the Ruelle operator. Among other things we analyze the case where temperature goes to zero and we show some selection results. Our general setting can be adapted in order to analyze the Thermodynamic Formalism for the Bernoulli space with countable infinite symbols. Moreover, the so called $X Y$ model also fits under our setting. In this last case $\mathrm{M}$ is the unitary circle $S^{1}$. We explore the differentiable structure of $\left(S^{1}\right)^{\mathbb{N}}$ by considering a certain class of smooth potentials and we show some properties of the corresponding main eigenfunctions.
\end{abstract}

\section{Introduction}

Let $\left(M, d_{M}\right)$ be a compact metric space. We consider the metric in $M^{\mathbb{N}}$ given by:

$$
d(x, y)=\sum_{n=1}^{\infty} \frac{1}{2^{n}} d_{M}\left(x_{n}, y_{n}\right),
$$

where $x=\left(x_{1}, x_{2}, \ldots\right)$ and $y=\left(y_{1}, y_{2}, \ldots\right)$. Note that $\mathcal{B}:=M^{\mathbb{N}}$ is compact by Tychonoff's theorem.

We denote by $H_{\alpha}$ the set of $\alpha$-Hölder functions $A: \mathcal{B} \rightarrow \mathbb{R}$ with the norm

$$
\|A\|_{\alpha}=\|A\|+|A|_{\alpha},
$$

where

$$
\|A\|=\sup _{x \in \mathcal{B}}|A(x)| \text { and }|A|_{\alpha}=\sup _{x \neq y} \frac{|A(x)-A(y)|}{d(x, y)^{\alpha}} .
$$

$\sigma: \mathcal{B} \rightarrow \mathcal{B}$ denotes the shift map which is defined by

$$
\sigma\left(x_{1}, x_{2}, x_{3}, \ldots\right)=\left(x_{2}, x_{3}, x_{4}, \ldots\right) .
$$


Let $\mathcal{C}$ be the space of continuous functions from $\mathcal{B}$ to $\mathbb{R}$, and we will fix an a-priori probability measure $\nu$ on the Borel sigma algebra over $M$. We assume that the support of $\nu$ is the set $M$. We stress the crucial point: $\nu$ needs to be a probability measure, not only a measure. Note that from our hypothesis if $x_{0}$ is isolated then $\nu\left(x_{0}\right)>0$.

For a fixed potential $A \in H_{\alpha}$ we define a Transfer Operator (also called Ruelle operator) $\mathcal{L}_{A}: \mathcal{C} \rightarrow \mathcal{C}$ by the rule

$$
\mathcal{L}_{A}(\varphi)(x)=\int_{M} e^{A(a x)} \varphi(a x) d \nu(a),
$$

where $x \in \mathcal{B}$ and $a x=\left(a, x_{1}, x_{2}, \ldots.\right)$ denote a pre-image of $x$ with $a \in M$.

We call One-dimensional Lattice System Theory this general setting. Rigorous mathematical results on Statistical Mechanics are presented in [49], 229], 34], [20, 22], [54, [1], [6], [32], 21] and [26].

We point out that a Holder potential $A$ defined on $M^{\mathbb{Z}}$ is coboundary with a potential in $M^{\mathbb{N}}$ (same proof as in [51]). In this way the Statistical Mechanics of interactions on $M^{\mathbb{Z}}$ can be understood via the analysis of the similar problem in $M^{\mathbb{N}}$.

In [3] it was investigated the Gibbs measure at positive and zero temperature for a potential $A$ (which depends on infinite coordinates) in the case $M=S^{1}$, where the a-priori measure is Lebesgue measure. This is the so called $X Y$ model (see 41, , 58, 24, which is considered in several applications to real problems in Physics. The spin in each site of the lattice is described by an angle from $[0,2 \pi)$. In the Physics literature, as far as we know, the potential $A$ depends on two coordinates. A well known example in applications is the potential $A(x)=A\left(x_{0}, x_{1}\right)=$ $\cos \left(x_{1}-x_{0}-\alpha\right)+\gamma \cos \left(2 x_{0}\right)$.

The present paper is a generalization of the setting presented in [3] for positive and zero temperature. We will also consider here a topic which was not addressed there, namely, the equilibrium (maximizing pressure) measure for the potential $A$.

There are several possible points of view for understanding Gibbs states in Statistical Mechanics (see [56], [53] for interesting discussions). We prefer the transfer operator method because we believe that the eigenfunctions and eigenprobabilities (which can be derived from the theory) allow a more deep understanding of the problem. For example, the information one can get from the main eigenfunction (defined in the whole lattice) is worthwhile, mainly in the limit when temperature goes to zero.

\section{Examples:}

Now we give a brief description of some other examples that fit in our setting. The last example will be explained in details in section 5 .

- If the alphabet is given by $M=\{1,2, \ldots, d\}$, and the a-priori measure is given by $\nu=$ $\frac{1}{d} \sum_{i=1}^{d} \delta_{i}$, then we have the original full shift in a finite set of $d$ symbols and the transfer operator is the classical Ruelle operator associated to a potential $A-\log (d)$ (see for example [51] and 37]). More precisely

$$
\mathcal{L}_{A}(\varphi)(x)=\int_{M} e^{A(a x)} \varphi(a x) d \nu(a)=\sum_{a \in\{1,2, \ldots, d\}} e^{A(a x)-\log (d)} \varphi(a x) .
$$

If we change the a-priori measure to $\nu=\sum_{i=1}^{d} p_{i} . \delta_{i}$, where $p_{i}>0$, and $\sum_{i=1}^{d} p_{i}=1$, then

$$
\mathcal{L}_{A}(\varphi)(x)=\sum_{a \in\{1,2, \ldots, d\}} e^{A(a x)} \varphi(a x) p_{a}=\sum_{a \in\{1,2, \ldots, d\}} e^{A(a x)+\log \left(p_{a}\right)} \varphi(a x)
$$

is the classical Ruelle operator with potential $A+\log (P)$, where $P\left(x_{1}, x_{2}, \ldots\right)=p_{x_{1}}$. 
- If $M_{0}=\left\{z_{i}, i \in \mathbb{N}\right\}$ is a countable infinite subset of $S^{1}$, where each point is isolated, and there is only one accumulating point $z_{\infty} \in S^{1} \backslash M_{0}$, then $M=M_{0} \cup\left\{z_{\infty}\right\}$ is a compact set. In this case $M$ can be identified with $\mathbb{N}$, where a special point $z_{\infty}$ plays the role of infinity (that is, a one-point compactification). We consider here the restricted distance we get from $S^{1}$ in $M$. If $\sum_{i \in \mathbb{N}} p_{i}=1$ with $p_{i} \geq 0$ and $\nu=\sum_{i \in \mathbb{N}} p_{i} \delta_{z_{i}}$ then $\nu$ is supported on the whole $M$, but $z_{\infty}$ is not an atom for $\nu$. The Thermodynamic Formalism with state space $\mathbb{N}$, or $\mathbb{Z}$, is considered for example in [55], [56], [19], , [36], [50], [33]. We will analyze in section a some of these results on the present setting.

Our main purpose here is to describe a general theory for the Statistical Mechanics of onedimensional spin lattices. We point out that most of the papers on the subject assume that the potential $A$ depends just on two (or, a finite number of) coordinates (as for instance is the case of [1], [6], [32]). We consider potentials which can depend on the infinite set of coordinates in $M^{\mathbb{N}}$.

In section 3 we consider the entropy, pressure and Variational Principle and its relations with eigenfunctions and eigenprobabilities of the Ruelle operator. This setting, as far as we know, was not considered before. In this case the entropy, by its very nature, is always a nonpositive number. If $M$ is not a finite set, invariant probabilities with support in a fixed point will have entropy equal to minus infinity. The infinite product of $d \nu$ on $M^{\mathbb{N}}$ will have zero entropy. We point out that, although at first glance, the fact that the entropy we define here is negative may look strange, our definition is the natural extension of the concept of Kolomogorov entropy. In the classical case, the entropy is positive because the a-priori measure is not a probability: is the counting measure.

Entropy and Pressure were considered before in other settings, as for instance in section II in [34] or [20]. In these works the authors consider a variational principle on boxes of finite length, and then they get the equilibrium as the limit probability on the lattice, when the size of the box goes to infinity. The concept of entropy was considered relative to a certain probability on the box (which in some sense plays the role of the a-priori probability). Our formalism is derived from the Ruelle operator point of view and is close to the approach described for instance in [51], where the probabilities are consider directly on $M^{\mathbb{N}}$. As we will see the concepts of entropy and the transfer operator are very much related. When the potential $A$ depends on an infinite number of coordinates in the lattice we believe our approach is more simple to state and to understand.

Other authors in previous works also considered Entropy and Transfer Operators on onedimensional Spin Lattices over metric spaces (see for instance section III 49], or section A3, or Proposition A4.9 in [39]), but we belive our approach is different.

Among other things we consider in section 1 the case where temperature goes to zero and show some selection results related with the Ergodic Optimization (see [18], 335], 16], 227, [40], [57]). Using the variational principle we obtain a simple proof of the fact that Gibbs states converge to maximizing measures when the temperature goes to zero (a question not discussed in [3]).

An important issue that does not appear in the classical Thermodynamic Formalism (in the sense of [51] and [37]) is the differentiable structure. We will show in section 6 that for a certain class of smooth potentials $A$ the associated main eigenfunction is also smooth.

\section{Ruelle operator}

Let $a^{n}$ be an element of $M^{n}$ having coordinates $a^{n}=\left(a_{n}, a_{n-1}, \ldots, a_{2}, a_{1}\right)$, we denote by $a^{n} x \in \mathcal{B}$ the concatenation of $a^{n} \in M^{n}$ with $x \in \mathcal{B}$, i.e., $a^{n} x=\left(a_{n}, \ldots, a_{1}, x_{1}, x_{2}, \ldots\right)$. In the case of $n=1$ we will write $a:=a^{1} \in M$, and $a x=\left(a, x_{1}, x_{2}, \ldots\right)$. 
The $n$-th iterate of $\mathcal{L}_{A}$ has the following expression

$$
\mathcal{L}_{A}^{n}(\varphi)(x)=\int_{M^{n}} e^{S_{n} A\left(a^{n} x\right)} \varphi\left(a^{n} x\right) d \nu^{n}\left(a^{n}\right),
$$

where $S_{n} A\left(a^{n} x\right)=\sum_{k=0}^{n-1} A\left(\sigma^{k}\left(a^{n} x\right)\right)$ and $d \nu^{n}\left(a^{n}\right)=\prod_{k=1}^{n} d \nu\left(a_{n-k+1}\right)$.

Let us show that $\mathcal{L}_{A}$ preserves the set of Hölder functions.

Lemma 1 If $\varphi \in H_{\alpha}$ then $\mathcal{L}_{A}(\varphi) \in H_{\alpha}$.

Proof: We have

$$
\frac{\left|\mathcal{L}_{A}(\varphi)(x)-\mathcal{L}_{A}(\varphi)(y)\right|}{d(x, y)^{\alpha}}=\frac{\left|\int_{M} e^{A(a x)} \varphi(a x) d \nu(a)-\int_{M} e^{A(a y)} \varphi(a y) d \nu(a)\right|}{d(x, y)^{\alpha}} .
$$

Now we use the fact that if $\varphi, A \in H_{\alpha}$, then $e^{A} \varphi \in H_{\alpha}$, and hence

$$
\frac{\int_{M}\left|e^{A(a x)} \varphi(a x)-e^{A(a y)} \varphi(a y)\right| d \nu(a)}{d(x, y)^{\alpha}} \leq \operatorname{Hol}\left(e^{A} \varphi\right) \nu(M)=\operatorname{Hol}\left(e^{A} \varphi\right) .
$$

Theorem 1 Consider a fixed a priori probability $\nu$. Let us fix $A \in H_{\alpha}$, then there exists a strictly positive Hölder eigenfunction $\psi_{A}$ for $\mathcal{L}_{A}: \mathcal{C} \rightarrow \mathcal{C}$ associated to a strictly positive eigenvalue $\lambda_{A}$. This eigenvalue is simple, which means the eigenfunction is unique (modulo multiplication by constant).

Proof: For each $0<s<1$, we define the operator $\mathcal{T}_{s, A}$ on $\mathcal{C}$, given by

$$
\mathcal{T}_{s, A}(u)(x)=\log \left(\int_{M} e^{A(a x)+s u(a x)} d \nu(a)\right) .
$$

The introduction of the parameter $s$ in the proof is an adaptation of an argument presented in [10] for the present setting.

An easy adaptation of the proof of the proposition 1 in [3] shows that $\mathcal{T}_{s, A}$ is an uniform contraction map. Let $u_{s}$ be the unique fixed point for $\mathcal{T}_{s, A}$, then $u_{s}$ satisfies

$$
\log \left(\int_{M} e^{A(a x)+s u_{s}(a x)} d \nu(a)\right)=u_{s}(x) .
$$

By the same arguments used in the proof of proposition 2 in [3], we can prove that the family $\left\{u_{s}\right\}_{0<s<1}$ is an equicontinuous family of functions. It follows from equation (1) that

$$
-\|A\|+s \min u_{s} \leq u_{s}(x) \leq\|A\|+s \max u_{s} .
$$

Hence, $-\|A\| \leq(1-s) \min u_{s} \leq(1-s) \max u_{s} \leq\|A\|$, for any $0<s<1$.

The family $\left\{u_{s}^{*}=u_{s}-\max u_{s}\right\}_{0<s<1}$ is equicontinuous and uniformly bounded. Let us fix a subsequence $s_{n} \rightarrow 1$ such that $\left[\left(1-s_{n}\right) \max u_{s_{n}}\right] \rightarrow k$, and that, using Arzela-Ascoli theorem, $\left\{u_{s_{n}}^{*}\right\}_{n \geq 1}$ has an accumulation point in $\mathcal{C}$, which we will call $u$.

Observe that for any $s$

$$
\begin{aligned}
e^{u_{s}^{*}(x)} & =e^{u_{s}(x)-\max u_{s}}=e^{-(1-s) \max u_{s}+u_{s}(x)-s \max u_{s}} \\
& =e^{-(1-s) \max u_{s}} \int_{M} e^{A(a x)+\left(s u_{s}(a x)-s \max u_{s}\right)} d \nu(a) .
\end{aligned}
$$


Taking limit where $n$ goes to infinity for the sequence $s_{n}$ we get that $u$ satisfies

$$
e^{u(x)}=e^{-k} \int_{M} e^{A(a x)+u(a x)} d \nu(a)=e^{-k} \mathcal{L}_{A}\left(e^{u}\right)(x) .
$$

This shows that $\psi_{A}:=e^{u}$ is a positive Holder eigenfunction for $\mathcal{L}_{A}$ associated to the eigenvalue $\lambda_{A}:=e^{k}$.

The proof of the uniqueness is exactly the same one presented in 3] (see comments after Theorem 3 in that paper).

The eigenfunction is unique up a multiplicative factor. There several ways to normalize it. We assume in this moment that the maximum of the eigenfunction is equal to 1.

We point out that is possible to generalize the above result for a priori probabilities which depend on the point $x \in M$. This will require some mild assumptions on this family of probabilities. We will not address this question here.

We say that a potential $B$ is normalized if $\mathcal{L}_{B}(1)=1$, which means it satisfies

$$
\int_{M} e^{B(a x)} d \nu(a)=1, \forall x \in \mathcal{B}
$$

In particular, $\forall x \in \mathcal{B}, a \rightarrow e^{B(a x)} d \nu(a)$ is a probability measure on $M$, and $\mathcal{L}_{B} u(x)$ can be seen as the expectation of the random variable $u$ with respect to this probability measure defined by the point $x$.

Let $A \in H_{\alpha}, \psi_{A}$ and $\lambda_{A}$ given by theorem 1, it is easy to see that

$$
\int_{M} \frac{e^{A(a x)} \psi_{A}(a x)}{\lambda_{A} \psi_{A}(x)} d \nu(a)=1, \forall x \in \mathcal{B} .
$$

Therefore we define the normalized potential $\bar{A}$ associated to $A$, as

$$
\bar{A}:=A+\log \psi_{A}-\log \psi_{A} \circ \sigma-\log \lambda_{A},
$$

where $\sigma: \mathcal{B} \rightarrow \mathcal{B}$ is the shift map. As $\psi_{A} \in H_{\alpha}$ we have that $\bar{A} \in H_{\alpha}$.

We define the Borel sigma-algebra $\mathcal{F}$ over $\mathcal{B}$ as the $\sigma$-algebra generated by the cylinders. By this we mean the sigma-algebra generated by sets of the form $B_{1} \times B_{2} \times \ldots \times B_{n} \times M^{\mathbb{N}}$, where $n \in \mathbb{N}$, and $B_{j}, j \in\{1,2, \ldots, n\}$, are open sets in $M$.

We say a probability measure $\mu$ over $\mathcal{F}$ is invariant, if for any Borel set $B$, we have that $\mu(B)=\mu\left(\sigma^{-1}(B)\right)$. We denote by $\mathcal{M}_{\sigma}$ the set of invariant probability measures.

We note that $\mathcal{B}$ is a compact metric space and by the Riesz Representation Theorem, a probability measure on the Borel sigma-algebra is identified with a positive linear functional $L: \mathcal{C} \rightarrow \mathbb{R}$ that sends the constant function 1 to the real number 1 . We also note that $\mu \in \mathcal{M}_{\sigma}$ if and only if, for any $\psi \in \mathcal{C}$ we have

$$
\int_{\mathcal{B}} \psi d \mu=\int_{\mathcal{B}} \psi \circ \sigma d \mu
$$

We define the dual operator $\mathcal{L}_{A}^{*}$ on the space of Borel measures on $\mathcal{B}$ as the operator that sends a measure $\mu$ to the measure $\mathcal{L}_{A}^{*}(\mu)$, defined by

$$
\int_{\mathcal{B}} \psi d \mathcal{L}_{A}^{*}(\mu)=\int_{\mathcal{B}} \mathcal{L}_{A}(\psi) d \mu
$$

for any $\psi \in \mathcal{C}$.

The next theorem is a generalization of propositions 4 and 5 of [3]. Here we consider $\mathcal{L}_{A}: H_{\alpha} \rightarrow H_{\alpha}$. 
Theorem 2 Let $A$ be a Hölder continuous potential, not necessarily normalized, $\psi_{A}$ and $\lambda_{A}$ the eigenfunction and eigenvalue given by the Theorem 1 . We associate to $A$ the normalized potential $\bar{A}=A+\log \psi_{A}-\log \psi_{A} \circ \sigma-\log \lambda_{A}$. Then

(a) there exists an unique fixed point $\mu_{A}$ for $\mathcal{L}_{\bar{A}}^{*}$, which is a $\sigma$-invariant probability measure;

(b) the measure

$$
\rho_{A}=\frac{1}{\psi_{A}} \mu_{A}
$$

satisfies $\mathcal{L}_{A}^{*}\left(\rho_{A}\right)=\lambda_{A} \rho_{A}$. Therefore, $\rho_{A}$ is an eigen-measure for $\mathcal{L}_{A}^{*}$;

(c) for any Hölder continuous function $w: \mathcal{B} \rightarrow \mathbb{R}$, we have that, in the uniform convergence topology,

$$
\mathcal{L}_{\bar{A}}^{n} \omega \rightarrow \int_{\mathcal{B}} \omega d \mu_{A}
$$

and

$$
\frac{\mathcal{L}_{A}^{n}(w)}{\left(\lambda_{A}\right)^{n}} \rightarrow \psi_{A} \int_{\mathcal{B}} w d \rho_{A}
$$

where $\mathcal{L}_{A}^{n}$ denotes the $n$-th iterate of the operator $\mathcal{L}_{A}: H_{\alpha} \rightarrow H_{\alpha}$.

Proof: (a) We begin by observing that the normalization property implies that the convex and compact set of Borel probability measures on $\mathcal{B}$ is preserved by the operator $\mathcal{L}_{\bar{A}}^{*}$. Therefore, using the Tychonoff-Schauder theorem we conclude the existence of a fixed point $\mu_{A}$ for the operator $\mathcal{L}_{\bar{A}}^{*}$. Now we prove that $\mu_{A}$ is $\sigma$-invariant: if $\psi \in \mathcal{C}$, we have

$$
\int_{\mathcal{B}} \psi \circ \sigma d \mu_{A}=\int_{\mathcal{B}} \psi \circ \sigma d \mathcal{L}_{\bar{A}}^{*}\left(\mu_{A}\right)=\int_{\mathcal{B}} \mathcal{L}_{\bar{A}}(\psi \circ \sigma) d \mu_{A}=\int_{\mathcal{B}} \psi d \mu_{A},
$$

where in the last equality we used the normalization hypothesis for $\bar{A}$. The uniqueness of the fixed point will be obtained in the proof of item (c).

(b) $\mathcal{L}_{\bar{A}}^{*}\left(\mu_{A}\right)=\mu_{A}$ implies that, for any $\psi \in \mathcal{C}$,

$$
\begin{aligned}
\int_{\mathcal{B}} \psi d \mu_{A} & =\int_{\mathcal{B}} \psi d \mathcal{L}_{\bar{A}}^{*}\left(\mu_{A}\right)=\int_{\mathcal{B}} \mathcal{L}_{\bar{A}}(\psi) d \mu_{A} \\
& =\int_{\mathcal{B}}\left(\int_{M} \psi(a x) \frac{e^{A(a x)} \psi_{A}(a x)}{\lambda_{A} \psi_{A}(x)} d \nu(a)\right) d \mu_{A}(x) .
\end{aligned}
$$

Now, if $\varphi \in \mathcal{C}$, making $\psi=\frac{\varphi}{\psi_{A}}$ in the last equation, we have

$$
\int_{\mathcal{B}} \frac{\varphi}{\psi_{A}} d \mu_{A}=\frac{1}{\lambda_{A}} \int_{\mathcal{B}}\left(\int_{M} \varphi(a x) \frac{e^{A(a x)}}{\psi_{A}(x)} d \nu(a)\right) d \mu_{A}(x),
$$

which is equivalent to

$$
\lambda_{A} \int_{\mathcal{B}} \varphi d \rho_{A}=\int_{\mathcal{B}} \mathcal{L}_{A}(\varphi) d \rho_{A}
$$

i.e., $\mathcal{L}_{A}^{*}\left(\rho_{A}\right)=\lambda_{A} \rho_{A}$.

(c) In order to prove item (c) we will need two claims. The first claim can be proved by induction.

First Claim: For any normalized Holder potential $B$, if $\|w\|$ denotes the uniform norm of the Holder function $w: \mathcal{B} \rightarrow \mathbb{R}$, we have

$$
\left|\mathcal{L}_{B}^{n}(w)(x)-\mathcal{L}_{B}^{n}(w)(y)\right| \leq\left[C_{e^{B}}\|w\|\left(\frac{1}{2^{\alpha}}+\ldots+\frac{1}{2^{n \alpha}}\right)+\frac{C_{w}}{2^{n \alpha}}\right] d(x, y)^{\alpha}
$$

where $C_{e^{B}}$ is the Holder constant of $e^{B}$ and $C_{w}$ is the Holder constant of $w$. 
As a consequence of the first claim, the set $\left\{\mathcal{L}_{\bar{A}}^{n} \omega\right\}_{n \geq 0}$ is equicontinuous. In order to prove that $\left\{\mathcal{L}_{\bar{A}}^{n} \omega\right\}_{n \geq 0}$ is uniformly bounded we use again the normalization condition, which implies $\left\|\overline{\mathcal{L}}_{A}^{n} \omega\right\| \leq\|w\|, \forall n \geq 1$.

Therefore, by the Arzela-Ascoli Theorem there exists an accumulation point, $\bar{\omega}$, for $\left\{\mathcal{L}_{\bar{A}}^{n} \omega\right\}_{n \geq 0}$, i.e., there exists a subsequence $\left\{n_{k}\right\}_{k \geq 0}$ such that

$$
\bar{\omega}(x)=\lim _{k \geq 0} \mathcal{L}_{\bar{A}}^{n_{k}} \omega(x) .
$$

Second Claim: $\bar{\omega}$ is a constant function.

To prove this claim, we begin by observing that

$$
\sup \bar{\omega} \geq \sup \mathcal{L}_{\bar{A}} \bar{\omega}
$$

(in fact this inequality holds for any function $w$ ). Now, (5) implies

$$
\bar{\omega}(x)=\lim _{k \geq 0} \mathcal{L}_{\bar{A}}^{n_{k}} \bar{\omega}(x),
$$

(possibly by a different subsequence) and this shows that what we have in (6) is indeed an equality: in fact, we have

$$
\sup \bar{\omega}=\sup \mathcal{L}_{\bar{A}}^{n} \bar{\omega} \forall n \geq 0 .
$$

Now, let $x_{M}^{n}$ be a maximum point of $\mathcal{L}_{\bar{A}}^{n} \bar{\omega}$, for any $n \geq 0$. We have

$$
\bar{\omega}\left(x_{M}^{0}\right)=\mathcal{L}_{\bar{A}}^{n} \bar{\omega}\left(x_{M}^{n}\right)
$$

and this proves the second claim, because the normalization property implies that $\mathcal{L}_{\bar{A}}^{n} \bar{\omega}\left(x_{M}^{n}\right)$ is a convex combination of $\bar{\omega}$ in the pre-images of $x_{M}^{n}$ (here we also use the fact that the support of the a-priori probability is the all space $M$ ).

Now that $\bar{\omega}$ is a constant function we can prove that

$$
\bar{\omega}=\int_{\mathcal{B}} \bar{\omega} d \mu_{A}=\lim _{k} \int_{\mathcal{B}} \mathcal{L}_{\bar{A}}^{n_{k}} \omega d \mu_{A}=\lim _{k} \int_{\mathcal{B}} \omega d\left(\mathcal{L}_{\bar{A}}^{*}\right)^{n_{k}}\left(\mu_{A}\right)=\int_{\mathcal{B}} \omega d \mu_{A},
$$

which shows that $\bar{\omega}$ does not depend on the subsequence chosen. Therefore, for any $x \in \mathcal{B}$ we have

$$
\mathcal{L}_{\bar{A}}^{n} \omega(x) \rightarrow \bar{\omega}=\int_{\mathcal{B}} \omega d \mu_{A} .
$$

The last limit shows that the fixed point $\mu_{A}$ is unique.

To finish the proof of item (c), as $A=\bar{A}-\log \psi_{A}+\log \psi_{A} \circ \sigma+\log \lambda_{A}$, we have

$$
S_{n} A(z)=\sum_{k=0}^{n-1} A \circ \sigma^{k}(z)=S_{n} \bar{A}(z)-\log \psi_{A}+\log \psi_{A} \circ \sigma^{n}+n \log \lambda_{A},
$$

and therefore

$$
\begin{aligned}
\frac{\mathcal{L}_{A}^{n}(w)(x)}{\lambda_{A}^{n}} & =\frac{1}{\lambda_{A}^{n}} \int_{M^{n}} e^{S_{n} A\left(a^{n} x\right)} w\left(a^{n} x\right) d \nu^{n}\left(a^{n}\right)=\psi_{A}(x) \int_{M^{n}} \frac{e^{S_{n} \bar{A}\left(a^{n} x\right)}}{\psi_{A}\left(a^{n} x\right)} w\left(a^{n} x\right) d \nu^{n}\left(a^{n}\right) \\
& =\psi_{A}(x) \mathcal{L}_{\bar{A}}^{n}\left(\frac{w}{\psi_{A}}\right) \rightarrow \psi_{A}(x) \int_{\mathcal{B}} \frac{w}{\psi_{A}} d \mu_{A}=\psi_{A}(x) \int_{\mathcal{B}} w d \rho_{A} .
\end{aligned}
$$

We call $\mu_{A}$ the Gibbs probability (or, Gibbs state) for $A$. We will leave the term equilibrium probability (or, equilibrium state) for the one which maximizes pressure. As we will see, this invariant probability measure over $\mathcal{B}$ describes the statistics in equilibrium for the 
interaction described by the potential $A$. The assumption that the potential is Hölder implies that the decay of iteration is fast.

We normalize $\psi_{A}$ by assuming that $\max \psi_{A}=1$. There are other possible normalizations. Therefore, $\rho_{A}$ is not a probability measure. From the item (c) above we conclude that

$$
\frac{\mathcal{L}_{A}^{n}(1)}{\left(\lambda_{A}\right)^{n}} \rightarrow \psi_{A} \rho_{A}(\mathcal{B}) .
$$

Proposition 1 The only Holder continuous eigenfunction $\psi$ of $\mathcal{L}_{A}$ which is totally positive is $\psi_{A}$.

Proof: Suppose $\psi: \mathcal{B} \rightarrow \mathbb{R}$ is a Holder continuous eigenfunction of $\mathcal{L}_{A}$ associated to some eigenvalue $\lambda$. It follows from item (c) of theorem 2 that

$$
\frac{\mathcal{L}_{A}^{n}(\psi)}{\lambda_{A}^{n}} \rightarrow \psi_{A} \int_{\mathcal{B}} \psi d \rho_{A} \text {, when } n \rightarrow \infty .
$$

Therefore, if $\psi>c>0$, then $\int_{\mathcal{B}} \psi d \rho_{A}>0$. Moreover, $\mathcal{L}_{A}^{n}(\psi)=\lambda^{n} \psi$. This is only possible if $\lambda=\lambda_{A}$ and $\psi=\psi_{A}$.

The next result follows from proposition 7 of [3].

Proposition 2 Suppose $\bar{A}$ is normalized, then the eigenvalue $\lambda_{\bar{A}}=1$ is maximal. Moreover, the remainder of the spectrum of $\mathcal{L}_{\bar{A}}: H_{\alpha} \rightarrow H_{\alpha}$ is contained in a disk centered at zero with radius strictly smaller than one.

The proof is the same one as the presented in [51] (see Theorem 2.2 (ii)).

We denote $\lambda_{\bar{A}}^{1}<\lambda_{\bar{A}}=1$ the spectral radius of $\mathcal{L}_{\bar{A}}$ when restricted to the set

$$
\left\{w \in H_{\alpha}: \int_{\mathcal{B}} w d \mu_{A}=0\right\}
$$

(which is the orthogonal complement of the space of constant functions, i.e., the orthogonal complement of the eigenspace associated to the maximal eigenvalue). One can also show the exponential decay of correlation for Hölder functions [3], which implies mixing and ergodic properties for $\mu_{A}$.

Proposition 3 If $v, w \in \mathcal{L}^{2}\left(\mu_{A}\right)$ are such that $w$ is Hölder and $\int_{\mathcal{B}} w d \mu_{A}=0$, then, there exists $C>0$ such that for all $n$

$$
\int_{\mathcal{B}}\left(v \circ \sigma^{n}\right) w d \mu_{A} \leq C\left(\lambda_{\bar{A}}^{1}\right)^{n} .
$$

In particular $\mu_{A}$ is mixing and therefore ergodic.

For the proof one can adapt Theorem 2.3 in [51.

\section{Entropy and Variational Principle}

In this section we will introduce a notion of entropy. Initially, this will be done only for Gibbs probabilities, and then we will extend this definition to invariant probabilities. After that we prove that the Gibbs probability obtained in the general setting above satisfies a variational principle. We will also study some general properties of this notion of entropy and compare it with the classical Kolmogorov entropy when $M=\{1, \ldots, d\}^{\mathbb{N}}$. Finally we will show that this definition is an extension of the notion of entropy for Markov measures (which are the Gibbs measures when the potential depends only on the first two coordinates), as introduced in [41].

Remember that $\mathcal{M}_{\sigma}$ is the set of invariant probability measures. 
Definition 1 Let $\nu$ be a fixed a-priori probability on $M$. We denote by $\mathcal{G}=\mathcal{G}_{\nu}$ the set of Gibbs measures, which means the set of $\mu \in \mathcal{M}_{\sigma}$, such that, $\mathcal{L}_{B}^{*}(\mu)=\mu$, for some normalized potential $B \in H_{\alpha}$. We define the entropy of $\mu \in \mathcal{G}$ as

$$
h(\mu)=h^{\nu}(\mu)=-\int_{\mathcal{B}} B(x) d \mu(x) .
$$

We will see below that $-\int_{\mathcal{B}} B d \mu$ is the infimum of

$$
\left\{-\int_{\mathcal{B}} A d \mu+\log \left(\lambda_{A}\right): A \in H_{\alpha}\right\}
$$

The above definition is different from the one briefly mentioned in section 3 in [3].

Remark: This concept of entropy depends on the choice of the a-priori measure $\nu$, which we choose to be a probability. In the classical case, when $M=\{1, \ldots, d\}$, the entropy $H(\mu)$ is computed with the a-priori measure $\nu$ given by $\sum_{j=1}^{d} \delta_{j}$ (which is not a probability). A comparison of the value of the above entropy $h(\mu)$, when $M=\{1, \ldots, d\}$, with the classical Kolmogorov entropy $H(\mu)$ (for the full shift) is discussed below, after proposition 7. For example, if the a-priori probability is $\nu=\frac{1}{d} \sum_{j=1}^{d} \delta_{j}$, to get the entropy $h(\mu)$ you just have to add $-\log d$ to the classical one $H(\mu)$. Therefore, in this particular case, the above definition results in a number between $-\log (d)$ and 0 . We point out that in the case $M$ has infinite cardinality the above definition $h(\mu)$ makes sense, is well defined, and it is the natural generalization of the previous concept.

Remark: Let $\mu$ be a Gibbs measure and $B$ the normalized potential associated to $\mu$, if we call $J=e^{-B}$, we have an equivalent definition of entropy given by

$$
h(\mu)=\int_{\mathcal{B}} \log (J(x)) d \mu(x) .
$$

We point out that $J=e^{-B}$ does not corresponds to the usual concept of Jacobian of the measure $\mu$. For example, consider a finite alphabet $M=\{1, \ldots, d\}$ and $\nu$ the a-priori probability given by $\nu(i)=p_{i}$, where $p_{i} \geq 0$ and $\sum_{i=1}^{d} p_{i}=1$. In this setting, the Ruelle operator is given by

$$
\mathcal{L}_{B} w(x)=\sum_{i=1}^{d} e^{B(i x)} w(i x) p_{i}
$$

which can be rewritten as

$$
\mathcal{L}_{B} w(x)=\sum_{i=1}^{d} e^{B(i x)+\log \left(p_{i}\right)} w(i x) .
$$

The last formulation fits in the classical thermodynamical formalism setting (see [51), for a potential $\tilde{B}(i x)=B(i x)+\log \left(p_{i}\right)$, where we know that the Jacobian (defined as $\lim _{n \rightarrow \infty} \frac{\mu\left[x_{1} x_{2} \ldots x_{n}\right]}{\mu\left[x_{2} x_{3} \ldots x_{n}\right]}$, when $\left[x_{1} x_{2} \ldots x_{n}\right]$ is the usual cylinder set) is given by $e^{-\tilde{B}}=e^{-B-\log \left(p_{i}\right)}$.

Proposition 4 If $\mu \in \mathcal{G}$, then we have $h(\mu) \leq 0$.

Proof: Let $\mu$ be a probability on $\mathcal{G}$ with associated normalized potential $B$. We have

$$
\begin{aligned}
h(\mu) & =-\int_{\mathcal{B}} B(x) d \mu(x)=\int_{\mathcal{B}} \log e^{-B(x)} d \mu(x) \leq \log \int_{\mathcal{B}} e^{-B(x)} d \mu(x) \\
& =\log \int_{\mathcal{B}} e^{-B(x)} d \mathcal{L}_{B}^{*}(\mu)(x)=\log \int_{\mathcal{B}} \mathcal{L}_{B} e^{-B(x)} d \mu(x)=0,
\end{aligned}
$$


where we have used Jensen's inequality and also $\mathcal{L}_{B} e^{-B(x)}=1$.

This negative entropy property will be useful in the next section to give an easy proof that the Gibbs measures of $\beta A$ select maximizing measures for $A$, when $\beta \rightarrow+\infty$. This result is obtained in the classical Thermodynamic Formalism Theory because the entropy is bounded. It will be also not difficult to get this in the present setting because the current notion of entropy is bounded above (by zero).

Now we state a lemma that will be used to prove the main result of this section, namely, the variational principle of Theorem 3. This lemma was shown to be true in the case $M$ is finite (and the classical Kolmogorov entropy) in 446.

Lemma 2 Let us fix a Hölder continuous potential $A$ and a measure $\mu \in \mathcal{G}$ with associated normalized potential $B$. We call $\mathcal{C}^{+}$the space of continuous positive functions on $\mathcal{B}$. We have

$$
h(\mu)+\int_{\mathcal{B}} A(x) d \mu(x)=\inf _{u \in \mathcal{C}^{+}}\left\{\int_{\mathcal{B}} \log \left(\frac{\mathcal{L}_{A} u(x)}{u(x)}\right) d \mu(x)\right\} .
$$

Proof: If we take $\tilde{u}(x)=e^{-A(x)+B(x)}$, then

$$
\log \left(\frac{\mathcal{L}_{A} \tilde{u}(x)}{\tilde{u}(x)}\right)=\log \left(\frac{\int_{M} e^{B(a x)} d \nu(a)}{e^{-A(x)+B(x)}}\right)=A(x)-B(x) .
$$

Integrating, we get

$$
\begin{aligned}
\int_{\mathcal{B}} \log \left(\frac{\mathcal{L}_{A} \tilde{u}(x)}{\tilde{u}(x)}\right) d \mu(x) & =\int_{\mathcal{B}} A(x) d \mu(x)-\int_{\mathcal{B}} B(x) d \mu(x) \\
& =h(\mu)+\int_{\mathcal{B}} A(x) d \mu(x) .
\end{aligned}
$$

Now, let us consider a general $\bar{u} \in \mathcal{C}^{+}$. Using the fact that $e^{-A+B}$ is a positive function, we can write $\bar{u}(x)=u(x) e^{-A(x)+B(x)}$. Note that, in this case,

$$
\mathcal{L}_{A} \bar{u}(x)=\int_{M} e^{B(a x)} u(a x) d \nu(a)=\mathcal{L}_{B} u(x) .
$$

Hence,

$$
\log \left(\frac{\mathcal{L}_{A} \bar{u}(x)}{\bar{u}(x)}\right)=\log \left(\mathcal{L}_{B} u(x)\right)-\log u(x)+A(x)-B(x),
$$

and therefore, by integration, we get

$$
\begin{aligned}
\int_{\mathcal{B}} \log \left(\frac{\mathcal{L}_{A} \bar{u}(x)}{\bar{u}(x)}\right) d \mu(x) & =\int_{\mathcal{B}} \log \left(\mathcal{L}_{B} u(x)\right) d \mu(x)-\int_{\mathcal{B}} \log u(x) d \mu(x) \\
& +\int_{\mathcal{B}} A(x) d \mu(x)-\int_{\mathcal{B}} B(x) d \mu(x) .
\end{aligned}
$$

Now, all we need to prove is that

$$
\int_{\mathcal{B}} \log \left(\mathcal{L}_{B} u(x)\right) d \mu(x)-\int_{\mathcal{B}} \log u(x) d \mu(x) \geq 0 .
$$

In order to do that, we use Jensen inequality, and we get $\log \left(\mathcal{L}_{B} u(x)\right) \geq \mathcal{L}_{B} \log u(x)$, which implies

$$
\int_{\mathcal{B}} \log \left(\mathcal{L}_{B} u(x)\right) d \mu(x) \geq \int_{\mathcal{B}} \mathcal{L}_{B} \log u(x) d \mu(x)=\int_{\mathcal{B}} \log u(x) d \mu(x),
$$

where we used $\mathcal{L}_{B}^{*}(\mu)=\mu$. 
Let $\mu \in \mathcal{G}$, with associated normalized potential $B, A \in H_{\alpha}$ and $\psi_{A}$ and $\lambda_{A}$, respectively, the positive eigenfunction and the maximal eigenvalue of $\mathcal{L}_{A}$, given by theorem 1 . From Lemma 2 we have

$$
\begin{aligned}
h(\mu)+\int_{\mathcal{B}} A d \mu & =\inf _{u \in \mathcal{C}^{+}}\left\{\int_{\mathcal{B}} \log \left(\frac{\mathcal{L}_{A} u(x)}{u(x)}\right) d \mu(x)\right\} \\
& \leq\left\{\int_{\mathcal{B}} \log \left(\frac{\mathcal{L}_{A} \psi_{A}}{\psi_{A}}\right) d \mu(x)\right\}=\log \lambda_{A} .
\end{aligned}
$$

This implies

$$
h(\mu)=-\int_{\mathcal{B}} B d \mu \leq-\int_{\mathcal{B}} A d \mu+\log \lambda_{A}, \quad \forall A \in H_{\alpha},
$$

with equality if $A=B\left(\right.$ as $\left.\lambda_{B}=1\right)$. Therefore

$$
h(\mu)=\inf _{A \in H_{\alpha}}\left\{-\int_{\mathcal{B}} A d \mu+\log \lambda_{A}\right\},
$$

with the minimum attained at $B$. Now, based on the last equation, we can extend the definition of entropy for all invariant measures.

Definition 2 Let $\mu$ be an invariant measure. We define the entropy of $\mu$ as

$$
h^{\nu}(\mu)=h(\mu)=\inf _{A \in H_{\alpha}}\left\{-\int_{\mathcal{B}} A d \mu+\log \lambda_{A}\right\},
$$

where $\lambda_{A}$ is the maximal eigenvalue of $\mathcal{L}_{A}$, given by theorem 1 .

This value is non positive and can be $-\infty$ as we will se later.

Definition 3 Given a Hölder potential $A$ we call the pressure of $A$ the value

$$
P(A)=\sup _{\mu \in \mathcal{M}_{\sigma}}\left\{h(\mu)+\int_{\mathcal{B}} A(x) d \mu(x)\right\} .
$$

A probability which attains such maximum value is called equilibrium state for $A$.

In the literature sometimes this value is called the Free Energy (see 21] for instance).

Now we will show the variational principle of pressure which characterizes the equilibrium state:

Theorem 3 (Variational Principle) Let $A \in H_{\alpha}$ be a Hölder continuous potential and $\lambda_{A}$ be the maximal eigenvalue of $\mathcal{L}_{A}$, then

$$
\log \lambda_{A}=P(A)=\sup _{\mu \in \mathcal{M}_{\sigma}}\left\{h(\mu)+\int_{\mathcal{B}} A(x) d \mu(x)\right\} .
$$

Moreover the supremum is attained on the Gibbs measure, i.e. the measure $\mu_{A}$ that satisfies $\mathcal{L}_{\bar{A}}^{*}\left(\mu_{A}\right)=\mu_{A}$.

Therefore, the Gibbs state and the equilibrium state for $A$ are given by the same measure $\mu_{A}$, which is the unique fixed point for the dual Ruelle operator associated to the normalized potential $\bar{A}$.

Proof: Consider a fixed $A \in H_{\alpha}$, by the definition of entropy, we have

$$
\begin{aligned}
& \sup _{\mu \in \mathcal{M}_{\sigma}}\left\{h(\mu)+\int_{\mathcal{B}} A(x) d \mu(x)\right\} \\
= & \sup _{\mu \in \mathcal{M}_{\sigma}}\left\{\inf _{B \in H_{\alpha}}\left\{-\int_{\mathcal{B}} B d \mu+\log \lambda_{B}\right\}+\int_{\mathcal{B}} A(x) d \mu(x)\right\} \\
\leq & \sup _{\mu \in \mathcal{M}_{\sigma}}\left\{-\int_{\mathcal{B}} A d \mu+\log \lambda_{A}+\int_{\mathcal{B}} A(x) d \mu(x)\right\}=\log \lambda_{A} .
\end{aligned}
$$


Hence,

$$
\log \lambda_{A} \geq \sup _{\mu \in \mathcal{M}_{\sigma}}\left\{h(\mu)+\int_{\mathcal{B}} A(x) d \mu(x)\right\} .
$$

On the order hand, as $A \in H_{\alpha}$, from theorem 10 we know that there exists $\lambda_{A}$ and $\varphi_{A}$, such that, $\mathcal{L}_{A}\left(\varphi_{A}\right)=\lambda_{A} \varphi_{A}$. Now, if we define $\bar{A}=A+\log \varphi_{A}-\log \varphi_{A} \circ \sigma-\log \lambda_{A}$, then, by Theorem 2, there exists a measure $\mu_{A}$ such that $\mathcal{L}_{\bar{A}}^{*}\left(\mu_{A}\right)=\mu_{A}$. This implies $\mu_{A} \in \mathcal{G}$, and

$$
h\left(\mu_{A}\right)=-\int_{\mathcal{B}} \bar{A}(x) d \mu_{A}(x)=-\int_{\mathcal{B}} A d \mu_{A}+\log \lambda_{A} .
$$

Therefore,

$$
\log \lambda_{A}=h\left(\mu_{A}\right)+\int_{\mathcal{B}} A d \mu_{A} \leq \sup _{\mu \in \mathcal{M}_{\sigma}}\left\{h(\mu)+\int_{\mathcal{B}} A(x) d \mu(x)\right\}
$$

In 41] a variational principle of pressure was considered. Other variational principles of pressure were described in [34 [49]. Our approach and also the kind of probabilities we consider are different of the ones in this last reference.

Theorem 4 (Pressure as Minimax) Given a Hölder potential A

$$
P(A)=\sup _{\mu \in \mathcal{M}_{\sigma}}\left[\inf _{u \in \mathcal{C}^{+}}\left\{\int_{\mathcal{B}} \log \left(\frac{\mathcal{L}_{A} u(x)}{u(x)}\right) d \mu(x)\right\}\right] .
$$

Proof: This follows at once from Lemma 2 (see also 45]).

It is known that periodic orbits can be used to get information about the pressure in the classical thermodynamic formalism setting, and also to approximate the equilibrium measure (see [51] chapter 5 and [44]). Therefore, the next corollary can be useful:

Corollary 1 For each $a^{n} \in M^{n}, a^{n}=\left(a_{n}, \ldots, a_{1}\right)$ let $a^{\infty} \in \mathcal{B}$ be the periodic orbit of period $n$ obtained by the successive concatenation of $a^{n}$, i.e., $a^{\infty}=\left(a_{n}, \ldots, a_{1}, a_{n}, \ldots, a_{1}, \ldots\right)$. Then,

$$
P(A)=\lim _{n \rightarrow \infty} \frac{1}{n} \log \left(\int_{M^{n}} e^{S_{n} A\left(a^{\infty}\right)} d \nu^{n}\left(a^{n}\right)\right) .
$$

Proof: Using Theorem 2 (c) and then the Variational Principle we conclude that for any fixed $x \in \mathcal{B}$

$$
\lim _{n \rightarrow \infty} \frac{1}{n} \log \left(\int_{M^{n}} e^{S_{n} A\left(a^{n} x\right)} d \nu^{n}\left(a^{n}\right)\right)=\lim _{n \rightarrow \infty} \frac{1}{n} \log \left(\mathcal{L}_{A}^{n}(1)(x)\right)=\log \left(\lambda_{A}\right)=P(A) .
$$

Using the fact that $A$ is Hölder continuous, there exists a constant $C>0$ such that

$$
\left|S_{n} A\left(a^{n} x\right)-S_{n} A\left(a^{\infty}\right)\right| \leq C\left(\frac{1}{2^{\alpha}}+\frac{1}{2^{2 \alpha}}+\ldots+\frac{1}{2^{n \alpha}}\right) d\left(x, a^{\infty}\right)^{\alpha} .
$$

Therefore, using that $\mathcal{B}$ is a compact set with finite diameter, there exists a constant $C>0$, such that,

$$
\left|S_{n} A\left(a^{n} x\right)-S_{n} A\left(a^{\infty}\right)\right| \leq C,
$$

for any $n \in \mathbb{N}, x \in \mathcal{B}$ and $a \in M$. Then,

$$
\int_{M^{n}} e^{S_{n} A\left(a^{n} x\right)-C} d \nu^{n}\left(a^{n}\right) \leq \int_{M^{n}} e^{S_{n} A\left(a^{\infty}\right)} d \nu^{n}\left(a^{n}\right) \leq \int_{M^{n}} e^{S_{n} A\left(a^{n} x\right)+C} d \nu^{n}\left(a^{n}\right),
$$


and

$$
\lim _{n \rightarrow \infty} \frac{1}{n} \log \left(\int_{M^{n}} e^{S_{n} A\left(a^{\infty}\right)} d \nu^{n}\left(a^{n}\right)\right)=\lim _{n \rightarrow \infty} \frac{1}{n} \log \left(\int_{M^{n}} e^{S_{n} A\left(a^{n} x\right)} d \nu^{n}\left(a^{n}\right)\right)=P(A) .
$$

Now we present a few properties of entropy:

Proposition 5 The entropy has the following properties:

a) $h(\mu) \leq 0$ for any invariant measure $\mu$.

b) $\nu^{\infty}=\nu \times \nu \times \nu \times \ldots$ has zero entropy.

c) The entropy is upper semi-continuous.

d) The entropy is a concave function in the space of invariant probabilities.

\section{Proof:}

a) We point out that $A=0$ is a normalized function, hence for any invariant measure $\mu$ we have $h(\mu) \leq 0$.

b) We are going to show that $\nu^{\infty}$ is the equilibrium measure for $A=0$. Indeed,

$$
\begin{aligned}
\int_{\mathcal{B}} g d \mathcal{L}_{A}^{*}\left(\nu^{\infty}\right) & =\int_{\mathcal{B}} \mathcal{L}_{A} g d \nu^{\infty}=\int_{\mathcal{B}} \int_{M} e^{0} g(a x) d \nu(a) d \nu^{\infty}(x) \\
& \stackrel{z=a x}{=} \int_{\mathcal{B}} g(z) d \nu^{\infty}(z) .
\end{aligned}
$$

which shows that $\mathcal{L}_{A}^{*}\left(\nu^{\infty}\right)=\nu^{\infty}$.

c) Fix an $\varepsilon>0$ and suppose $\mu_{n}$ converges to $\mu$. By definition of $h(\mu)$, we can choose $A \in H_{\alpha}$ such that

$$
-\int_{\mathcal{B}} A d \mu+\log \lambda_{A} \leq h(\mu)+\varepsilon .
$$

If $n$ is large enough, we have $\left|\int_{\mathcal{B}} A d \mu_{n}-\int_{\mathcal{B}} A d \mu\right|<\varepsilon$. Then,

$$
h\left(\mu_{n}\right) \leq-\int_{\mathcal{B}} A d \mu_{n}+\log \lambda_{A} \leq-\int_{\mathcal{B}} A d \mu+\log \lambda_{A}+\varepsilon \leq h(\mu)+2 \varepsilon,
$$

therefore $\lim \sup h\left(\mu_{n}\right) \leq h(\mu)+2 \varepsilon$.

d) Let $\mu_{1}$ and $\mu_{2}$ be $\sigma$-invariant probabilities, $\varepsilon \in(0,1)$ and $\mu=\varepsilon \mu_{1}+(1-\varepsilon) \mu_{2}$. Then

$$
\begin{aligned}
& h\left(\varepsilon \mu_{1}+(1-\varepsilon) \mu_{2}\right)=h(\mu)=\inf _{A}\left(-\int_{\mathcal{B}} A d \mu+\log \lambda_{A}\right) \\
& =\inf _{A}\left(-\varepsilon \int_{\mathcal{B}} A d \mu_{1}+(1-\varepsilon) \int_{\mathcal{B}} A d \mu_{2}+\log \lambda_{A}\right) \\
& \geq \inf _{A}\left(-\varepsilon \int_{\mathcal{B}} A d \mu_{1}+\varepsilon \cdot \log \lambda_{A}\right)+\inf _{A}\left(-(1-\varepsilon) \int_{\mathcal{B}} A d \mu_{2}+(1-\varepsilon) \cdot \log \lambda_{A}\right) \\
& =\varepsilon h\left(\mu_{1}\right)+(1-\varepsilon) h\left(\mu_{2}\right) .
\end{aligned}
$$

Remark: The entropy of a probability measure supported on periodic orbit can be $-\infty$. Indeed, suppose $M=[0,1]$, and $A_{c}: M^{\mathbb{N}} \rightarrow \mathbb{R}$ given by $A_{c}(x)=\log \left(\frac{c}{1-e^{-c}} e^{-c x_{1}}\right)$. Suppose 
the a-priori $\nu$ measure is the Lebesgue measure. We have that for each $c>0$, the function $A_{c}$ is a $C^{1}$ normalized potential (therefore belongs to $H_{\alpha}$ ), which depends only on the first coordinate of $x$. Note that $\mathcal{L}_{A_{c}}(1)=1$. Let $\mu$ be the Dirac Measure on $0^{\infty}$. We have $h(\mu) \leq-\int_{\mathcal{B}} A_{c} d \mu=-A_{c}\left(0^{\infty}\right)=-\log \left(\frac{c}{1-e^{-c}}\right) \rightarrow-\infty$ when $c \rightarrow \infty$. This shows that $h(\mu)=-\infty$. An easy adaptation of the arguments can be done to prove that, in this setting, invariant measures supported on periodic orbits have entropy $-\infty$.

Note the subtle point that the entropy depends on the a-priori probability and moreover all subsequent concepts we introduced, like for example the Ruelle operator,

$$
\mathcal{L}_{A}(\varphi)(x)=\int_{M} e^{A(a x)} \varphi(a x) d \nu(a)
$$

assume conditions on the pre-images of $\sigma$. Therefore, given an iterate $\sigma^{n}$, if one wants to consider the entropy of a $\sigma^{n}$-invariant probability, then we need to specify a certain a-priori probability. We will address this question now.

Entropy of iterates: Suppose $M^{n}$ is the compact set given by

$$
\left\{\left(x_{1}, x_{2}, \ldots, x_{n}\right) \mid x_{i} \in M, \forall 1 \leq i \leq n\right\},
$$

with the sum or the maximum norm. Let $\sigma^{n}$ be the shift map defined on the Bernoulli space given by $\mathcal{B}^{n} \equiv\left(M^{n}\right)^{\mathbb{N}}$. We know that $\sigma^{n}$ is the $n$-th iterate of $\sigma$ in the original Bernoulli space, but we prefer to see $\sigma^{n}$ as a new map defined on a new Bernoulli space. If we do that, all the theory developed above applies to $\sigma^{n}$, we have a Ruelle operator with an a-priori measure given by $\nu^{n}$, and therefore the entropy of a Gibbs measure to the new map $\sigma^{n}$ is well defined. Note that the new Bernoulli set $\mathcal{B}^{n}$ can be identified with the original $\mathcal{B}$ and an invariant measure for $\sigma$ is also an invariant measure for $\sigma^{n}$.

Proposition 6 If $\mathcal{G}^{n}$ denotes the set of Gibbs measures on $\mathcal{B}^{n}$, then $\mu \in \mathcal{G}$ implies $\mu \in \mathcal{G}^{n}$ and

$$
h_{\mu}^{\nu^{n}}\left(\sigma^{n}\right)=h_{\mu}\left(\sigma^{n}\right)=n h_{\mu}(\sigma)=n h_{\mu}^{\nu}(\sigma) .
$$

Proof: Note that if $\mu$ is a $\sigma$-invariant measure, then $\mu$ is invariant for $\sigma^{n}$. Also, if $B \in H_{\alpha}$ is a normalized potential for $\sigma$, then the Birkhoff sum $B^{n} \equiv \sum_{j=0}^{n-1} B \circ \sigma^{j}$ is a normalized potential for the map $\sigma^{n}$. Let us first prove that if $\mu$ is the Gibbs measure for the Ruelle operator associated to $B$, then, $\mu$ ( which is indeed a measure on $\mathcal{B}^{n}$ ), is also Gibbs for $B^{n}$. In order to do that, note that

$$
\begin{aligned}
& \mathcal{L}_{B^{n}}^{m}(\varphi)(x)=\int_{\left(M^{n}\right)^{m}} e^{S_{m} B^{n}\left(a^{n m} x\right)} \varphi\left(a^{n m} x\right) d\left(\nu^{n}\right)^{m}\left(a^{n m}\right) \\
& =\int_{\left(M^{n}\right)^{m}} e^{S_{m n} B\left(a^{n m} x\right)} \varphi\left(a^{n m} x\right) d \nu^{n m}\left(a^{n m}\right)=\mathcal{L}_{B}^{m n}(\varphi)(x) \rightarrow \int_{\mathcal{B}} \varphi d \mu .
\end{aligned}
$$

Now the $\sigma^{n}$-entropy of $\mu$, given by the integral of the $B^{n}$, equals $n$ times the $\sigma$-entropy of $\mu$, because, using the fact that $\mu$ is $\sigma$-invariant, we have

$$
\begin{aligned}
& h_{\mu}\left(\sigma^{n}\right)=-\int_{\mathcal{B}^{n}} B^{n} d \mu=-\int_{\mathcal{B}^{n}} \sum_{j=0}^{n-1} B \circ \sigma^{j} d \mu \\
= & -\sum_{j=0}^{n-1} \int_{\mathcal{B}} B \circ \sigma^{j} d \mu=-n \int_{\mathcal{B}} B d \mu=n h_{\mu}(\sigma) .
\end{aligned}
$$




\section{Relations with Kolmogorov Entropy:}

Let us consider the construction of the entropy by partitions method, in the case $M$ is finite. We begin by remembering that, by the Kolmogorov-Sinai Theorem, the classical entropy of $\mu$, which we will denote by $H(\mu)$, is given by

$$
H(\mu)=\lim _{n \rightarrow \infty}-\frac{1}{n} \sum_{i_{1}, \ldots, i_{n}} \mu\left(\left[i_{1} \ldots i_{n}\right]\right) \log \left(\mu\left(\left[i_{1} \ldots i_{n}\right]\right)\right) .
$$

Proposition 7 Let $M=\{1, \ldots, d\}$ and $\nu=\sum_{i=1}^{d} p_{i} \delta_{i}$ be the a-priori probability on $M$. For any Gibbs measure $\mu$ :

(a)

$$
H(\mu)=h^{\nu}(\mu)-\sum_{i=1}^{d} \log \left(p_{i}\right) \cdot \mu([i])
$$

(b)

$$
h^{\nu}(\mu)=-\lim _{n \rightarrow \infty} \frac{1}{n} \sum_{i_{1}, \ldots, i_{n}} \mu\left(\left[i_{1} \ldots i_{n}\right]\right) \log \left(\frac{\mu\left(\left[i_{1} \ldots i_{n}\right]\right)}{p_{i_{1}} \ldots p_{i_{n}}}\right)
$$

where

$$
\left[i_{1} \ldots i_{n}\right]=\left\{x \in M^{\mathbb{N}}: x_{1}=i_{1}, \ldots, x_{n}=i_{n}\right\}
$$

Proof:

If $\mu$ is a Gibbs measure, there exists a normalized potential $A$ associated to $\mu$, which implies

$$
\int_{M} e^{A(a x)} d \nu(a)=\sum_{i=1}^{d} e^{A(i x)} p_{i}=1, \quad \forall x \in M^{\mathbb{N}},
$$

which is equivalent to

$$
\sum_{i=1}^{d} e^{A(i x)+\log \left(p_{i}\right)}=1, \quad \forall x \in M^{\mathbb{N}}
$$

Moreover, $\mathcal{L}_{A}^{*}(\mu)=\mu$ implies that $\mu$ is a fixed point for the Classical Ruelle Operator with the normalized potential $A+\log (P)$, where $P\left(y_{1}, y_{2}, \ldots\right)=p_{y_{1}}$. Therefore

$$
H(\mu)=-\int_{\mathcal{B}} A+\log (P) d \mu=h^{\nu}(\mu)-\int_{\mathcal{B}} \log (P) d \mu=h^{\nu}(\mu)-\sum_{i=1}^{d} \log \left(p_{i}\right) \cdot \mu([i]) .
$$

which ends the proof of item (a)

In order to prove item (b), we note that, from the last equation, and using that $\mu$ is an $\sigma$-invariant measure, we have, for any $n \geq 1$

$$
\begin{aligned}
H(\mu) & =h^{\nu}(\mu)-\frac{1}{n} \int_{\mathcal{B}} \log (P)+\ldots+\log \left(P \circ \sigma^{n-1}\right) d \mu \\
& =h^{\nu}(\mu)-\frac{1}{n} \int_{\mathcal{B}} \log \left(p_{x_{1}} \ldots p_{x_{n}}\right) d \mu(x) \\
& =h^{\nu}(\mu)-\frac{1}{n} \sum_{i_{1}, \ldots, i_{n}} \mu\left(\left[i_{1} \ldots i_{n}\right]\right) \log \left(p_{i_{1}} \ldots p_{i_{n}}\right) .
\end{aligned}
$$


Then,

$$
\begin{aligned}
h^{\nu}(\mu) & =H(\mu)+\frac{1}{n} \sum_{i_{1}, \ldots, i_{n}} \mu\left(\left[i_{1} \ldots i_{n}\right]\right) \log \left(p_{i_{1}} \ldots p_{i_{n}}\right) \\
& =H(\mu)+\lim _{n \rightarrow \infty} \frac{1}{n} \sum_{i_{1}, \ldots, i_{n}} \mu\left(\left[i_{1} \ldots i_{n}\right]\right) \log \left(p_{i_{1}} \ldots p_{i_{n}}\right) \\
& =-\lim _{n \rightarrow \infty} \frac{1}{n} \sum_{i_{1}, \ldots, i_{n}} \mu\left(\left[i_{1} \ldots i_{n}\right]\right) \log \left(\frac{\mu\left(\left[i_{1} \ldots i_{n}\right]\right)}{p_{i_{1}} \ldots p_{i_{n}}}\right) .
\end{aligned}
$$

where in the last equation we used (8).

In particular, it follows from item (a) above that, when $p_{i}=\frac{1}{d}$, for all $i$, we have

$$
h^{\nu}(\mu)=H(\mu)-\log (d) .
$$

The above proposition can be interpreted in the following way: in the classical definition of Kolmogorov entropy it is considered the a-priori measure $\nu=\sum_{i=1}^{\infty} \delta_{i}$ on $M$, which is not a probability.

\section{Markov Chains with values on $S^{1}$ :}

Now we recall the concept of Markov measures and show that the entropy defined above is an extension of the concept of entropy for Markov measures, as introduced in [41].

Let $K: M^{2} \rightarrow \mathbb{R}, \theta: M \rightarrow \mathbb{R}$, satisfying

$$
\int_{M} K\left(x_{1}, x_{2}\right) d \nu\left(x_{2}\right)=1, \forall x_{1} \text { and } \int_{M} \theta\left(x_{1}\right) K\left(x_{1}, x_{2}\right) d \nu\left(x_{1}\right)=\theta\left(x_{2}\right), \forall x_{2} .
$$

We call $K$ a transition kernel and $\theta$ the stationary measure for $K$. As in 41, we define the absolutely continuous Markov measure associated to $K$ and $\theta$, as

$$
\mu\left(A_{1} \ldots A_{n} \times M^{\mathbb{N}}\right):=\int_{A_{1} \ldots A_{n}} \theta\left(x_{1}\right) K\left(x_{1}, x_{2}\right) \ldots K\left(x_{n-1}, x_{n}\right) d \nu\left(x_{n}\right) \ldots d \nu\left(x_{1}\right),
$$

for any cylinder $A_{1} \ldots A_{n} \times M^{\mathbb{N}}$.

The next proposition show us the importance of a.c. Markov measures:

Proposition 8 We will show that

a) Given a Hölder continuous potential $A\left(x_{1}, x_{2}\right)$ (not necessarily normalized) depending on two coordinates, there exists a Markov measure that is Gibbs for A.

b) The converse is also true: given an absolutely continuous Markov measure defined by $K$ and $\theta$, there exists a certain Hölder continuous normalized potential $A\left(x_{1}, x_{2}\right)$, such that the Markov measure defined by $\theta$ and $K$ is the Gibbs measure for A.

Therefore, any a.c. Markov measure is Gibbs for a potential depending on two variables, and conversely, any potential depending on two variables has a Gibbs measure which is an a.c. Markov Measure.

In other words, if we restrict our analysis to potentials that depend just on the first two coordinates, we have that the set of a.c. Markov Measures coincides with the set of Gibbs measures.

\section{Proof:}

(a) Given a potential $A\left(x_{1}, x_{2}\right)$, non-normalized, as in 41 define $\theta_{A}: M \rightarrow \mathbb{R}$ by

$$
\theta_{A}\left(x_{1}\right):=\frac{\psi_{A}\left(x_{1}\right) \bar{\psi}_{A}\left(x_{1}\right)}{\pi_{A}},
$$


and a transition $K_{A}: M^{2} \rightarrow \mathbb{R}$ by

$$
K_{A}\left(x_{1}, x_{2}\right):=\frac{e^{A\left(x_{1}, x_{2}\right)} \bar{\psi}_{A}\left(x_{2}\right)}{\bar{\psi}_{A}\left(x_{1}\right) \lambda_{A}},
$$

where $\psi_{A}$ and $\bar{\psi}_{A}$ are the eigenfunctions associated to the maximal eigenvalue $\lambda_{A}$ of the operators

$$
L_{A} \psi\left(x_{2}\right)=\int_{M} e^{A\left(x_{1}, x_{2}\right)} \psi\left(x_{1}\right) d \nu\left(x_{1}\right) \quad \text { and } \quad \bar{L}_{A} \psi\left(x_{1}\right)=\int_{M} e^{A\left(x_{1}, x_{2}\right)} \psi\left(x_{2}\right) d \nu\left(x_{2}\right)
$$

and $\pi_{A}=\int_{M} \psi_{A}\left(x_{1}\right) \bar{\psi}_{A}\left(x_{1}\right) d \nu\left(x_{1}\right)$.

Then, by the same arguments used to prove theorem 16 of [3], we obtain that the Markov measure $\mu_{A}$ defined by (10) (considering $K_{A}$ and $\theta_{A}$ ) is Gibbs for $A$, i.e. a fixed point for the dual Ruelle operator $\mathcal{L}_{\bar{A}}^{*}$, where $\bar{A}=A+\log \psi_{A}\left(x_{1}\right)-\log \psi_{A}\left(x_{2}\right)-\log \lambda_{A}$.

(b) Let $K$ and $\theta$ satisfying (9), and define $A=\log K$, we have $\bar{L}_{A}(1)=1$ which implies $\lambda_{A}=1$ and $\bar{\psi}_{A}=1$. Let $\psi_{A}$ be maximal eigenfunction for $L_{A}$.

Using (12), we get $K_{A}\left(x_{1}, x_{2}\right)=e^{A\left(x_{1}, x_{2}\right)}=K\left(x_{1}, x_{2}\right)$. Define $\theta_{A}=\frac{\psi_{A}}{\pi_{A}}$. We have that $\theta_{A}$ is an invariant density for $K$, therefore $\theta_{A}=\theta$. Then, also by theorem 16 page of [3] , we have that the Markov measure defined by $K$ and $\theta$ is Gibbs for $A$.

Next proposition shows that the concept of entropy introduced in $⿴$ 目 is a generalization of the concept of entropy defined in [41, which could only be applied to a.c. Markov measures:

Proposition 9 Let $\mu$ be the Markov measure defined by a transition kernel $K$ and a stationary measure $\theta$, given in (10). The definition of entropy given in [41]:

$$
S(\theta K)=-\int_{M^{2}} \theta\left(x_{1}\right) K\left(x_{1}, x_{2}\right) \log \left(K\left(x_{1}, x_{2}\right)\right) d \nu\left(x_{1}\right) d \nu\left(x_{2}\right) \leq 0
$$

coincides with the present definition 国.

Proof: As in the proof of proposition \&, note that the normalized potential associated to $A(x, y)=\log K(x, y)$ is

$$
\bar{A}\left(x_{1}, x_{2}\right)=\log K\left(x_{1}, x_{2}\right)+\log \psi_{A}\left(x_{1}\right)-\log \psi_{A}\left(x_{2}\right),
$$

where $\psi_{A}$ is the maximal eigenfunction of the operator $L_{A}$. Note also that $\bar{A}$ depends only on the first two coordinates.

Let $\mu$ be the Gibbs measure associated to $\bar{A}$, hence by definition 2 we have

$$
\begin{aligned}
h(\mu) & =-\int_{\mathcal{B}} \bar{A}\left(x_{1}, x_{2}\right) d \mu(x)=-\int_{\mathcal{B}} \log K\left(x_{1}, x_{2}\right) d \mu(x) \\
& =\int_{M^{2}} \log K\left(x_{1}, x_{2}\right) \theta\left(x_{1}\right) K\left(x_{1}, x_{2}\right) d \nu\left(x_{1}\right) d \nu\left(x_{2}\right)=S(\theta K) .
\end{aligned}
$$

\section{Zero temperature}

Consider a fixed Hölder potential $A$ and a real variable $\beta>0$. We denote, respectively, by $\psi_{\beta A}$ and $\mu_{\beta A}$, the eigenfunction for the Ruelle operator associated to $\beta A$ and the equilibrium measure (Gibbs) for $\beta A$. We would like to investigate general properties of the limits of $\mu_{\beta_{n} A}$ and of $\frac{1}{\beta_{n}} \log \psi_{\beta_{n} A}$ when $\beta_{n} \rightarrow \infty$. Some results of this section are generalizations of the ones in [3]. It is well known that the parameter $\beta$ represents the inverse of the temperature. 
It is fair to call "Gibbs state at zero temperature for the potential $A$ " any of the weak limits of convergent subsequences $\mu_{\beta_{n} A}$. Even when the potential $A$ is Hölder, Gibbs state at zero temperature do not have to be unique. In the case there exist the weak limit $\mu_{\beta A} \rightarrow \mu$, $\beta \rightarrow \infty$, we say that there exists selection of Gibbs state for $A$ at temperature zero.

Remark : Given $\beta$ and $A$, the Hölder constant of $u_{\beta A}=\log \left(\psi_{\beta A}\right)$, depends on the Hölder constant for $\beta A$, and is given by $\beta \frac{2^{\alpha}}{2^{\alpha}-1} H_{o l}$ (see [3]). As we normalize $\psi_{\beta A}$ assuming that $\max \psi_{\beta A}=1$, the family of functions $\frac{1}{\beta} \log \left(\psi_{\beta A}\right), \beta>0$, is uniformly bounded. Note that when we normalize $\psi_{\beta A}$ the Hölder constant of $\log \left(\psi_{\beta A}\right)$ remains unchanged, which assures the family $\frac{1}{\beta} \log \left(\psi_{\beta A}\right), \beta>0$, is equicontinuous.

Therefore, there exists a subsequence $\beta_{n} \rightarrow \infty$, and $V$ Holder, such that, on the uniform convergence topology

$$
V:=\lim _{n \rightarrow \infty} \frac{1}{\beta_{n}} \log \left(\psi_{\beta_{n} A}\right) .
$$

Remember that we denote by $\mathcal{M}_{\sigma}$ the set of $\sigma$ invariant Borel probability measures over $\mathcal{B}$. As $\mathcal{M}_{\sigma}$ is compact, given $A$, there always exists a subsequence $\beta_{n}$, such that $\mu_{\beta_{n} A}$ converges to an invariant probability measure.

The limits of $\mu_{\beta A}$ are related (see below) with the following problem: given $A: \mathcal{B} \rightarrow \mathbb{R}$ Holder, we want to find probabilities that maximize, over $\mathcal{M}_{\sigma}$, the value

$$
\int_{\mathcal{B}} A(x) d \mu(\mathbf{x})
$$

We define

$$
m(A)=\max _{\mu \in \mathcal{M}_{\sigma}}\left\{\int_{\mathcal{B}} A d \mu\right\} .
$$

Any of the probability measures which attains the maximal value will be called a maximizing probability measure, which will be sometimes denoted generically by $\mu_{\infty}$. As $\mathcal{M}_{\sigma}$ is compact, there exist always at least one maximizing probability measure. It is also true that there exists ergodic maximizing probability measures. Indeed, the set of maximizing probability measures is convex, compact and the extreme probability measures of this convex set are ergodic (can not be expressed as convex combination of others [37]). Any maximizing probability measure is a convex combination of ergodic ones [52]. Results obtained in this setting belong to what is called Ergodic Optimization Theory [35].

The possible limits of $\frac{1}{\beta_{n}} \log \psi_{\beta_{n} A}$ are related (see below) with the following concept:

Definition $4 A$ continuous function $u: \mathcal{B} \rightarrow \mathbb{R}$ is called a calibrated subaction for $A: \mathcal{B} \rightarrow \mathbb{R}$, if, for any $y \in \mathcal{B}$, we have

$$
u(y)=\max _{\sigma(x)=y}[A(x)+u(x)-m(A)] .
$$

This can also be expressed as

$$
m(A)=\max _{a \in M}\{A(a y)+u(a y)-u(y)\} .
$$

Note that for any $x \in \mathcal{B}$ we have

$$
u(\sigma(x))-u(x)-A(x)+m(A) \geq 0 .
$$

The above equation for $u$ can be seen as a kind of discrete version of the concept of subsolution of the Hamilton-Jacobi equation [15] [5] [23]. It can be also seen as a kind of dynamic additive eigenvalue problem [1] [12] 228.

We note that $m(A)$ can be characterized by

$$
m(A)=\inf \{\gamma: \exists u \in C, \gamma+u \circ \sigma-u-A \geq 0\},
$$


where $\mathcal{C}$ denotes the set of continuous real-valued functions. In some sense this corresponds to the dual problem in transport theory [43. Any invariant measure help us to estimate $m(A)$ from below. The continuous functions on the dual problem help us to estimate $m(A)$ from above.

If $u$ is a calibrated subaction, then $u+c$, where $c$ is a constant, is also a calibrated subaction. An interesting question is when such calibrated subaction $u$ is unique up to an additive constant (see [4] and [27).

Remember that if $\mu$ is $\sigma$-invariant, then for any continuous function $u: \mathcal{B} \rightarrow \mathbb{R}$ we have

$$
\int_{\mathcal{B}}[u(\sigma(x))-u(x)] d \mu=0 .
$$

Therefore if $\mu_{\infty}$ is a maximizing probability measure for $A$ and $u$ is a calibrated subaction for $A$, then (see for instance [16] [35] [57] for a similar result) for any $x$ in the support of $\mu_{\infty}$, we have

$$
u(\sigma(x))-u(x)-A(x)+m(A)=0 .
$$

In this way if we know the value $m(A)$, then a calibrated subaction $u$ for $A$ can help us to identify the support of maximizing probabilities. The above equation can be eventually true outside the union of the supports of the maximizing probabilities (see an interesting example due to R. Leplaideur [17).

We show below that if there exists a subsequence $\beta_{n} \rightarrow \infty$, such that on the uniform convergence

$$
V:=\lim _{n \rightarrow \infty} \frac{1}{\beta_{n}} \log \left(\psi_{\beta_{n} A}\right),
$$

then such $V$ is a calibrated subaction for $A$. When there exists a $V$ which is the limit

$$
V:=\lim _{\beta \rightarrow \infty} \frac{1}{\beta} \log \left(\psi_{\beta A}\right)
$$

(not just via a subsequence) we say we have selection of subaction at temperature zero. Positive results in this direction are presented in [4], [42] and [41].

There exists here a subtle point. Sub-action is a concept in Ergodic Optimization and does not depend on the existence of an a-priori probability $\nu$ in $M$. On the other hand, the eigenfunction $\psi_{\beta_{n} A}$ is associated to a Ruelle Operator, which depends on the a-priori measure. In any case, for any given a-priori probability $\nu$, if the associated family of eigenfunctions $\psi_{\beta_{n} A}$ converges, it will converge to a sub-action for $A$.

Lemma 3 For any $\beta$, we have $-\|A\|<\frac{1}{\beta} \log \lambda_{\beta}<\|A\|$.

Proof: Fix $\beta>0$. Let $\bar{x}$ be the maximum of $\psi_{\beta A}$ in $\mathcal{B}$ and $\tilde{x}$ be the minimum of $\psi_{\beta A}$ in $\mathcal{B}$. If $\|A\|$ is the uniform norm of $A$, we have

$$
\lambda_{\beta}=\frac{1}{\psi_{\beta A}(\bar{x})} \int_{M} e^{\beta A(a \bar{x})} \psi_{\beta A}(a \bar{x}) d \nu(a) \leq \int_{M} e^{\beta A(a \bar{x})} d \nu(a) \leq e^{\beta\|A\|},
$$

and

$$
\lambda_{\beta}=\frac{1}{\psi_{\beta A}(\tilde{x})} \int_{M} e^{\beta A(a \tilde{x})} \psi_{\beta A}(a \tilde{x}) d \nu(a) \geq \int_{M} e^{\beta A(a \bar{x})} d \nu(a) \geq e^{-\beta\|A\|}
$$

The next result can be seen like a kind of measure theoretical version of the Laplace's method.

Lemma 4 Suppose $W_{t}: M \rightarrow \mathbb{R}$ converges uniformly to $W: M \rightarrow \mathbb{R}$, when $t \rightarrow+\infty$. Then

$$
\lim _{t \rightarrow \infty} \frac{1}{t} \log \int_{M} e^{t W_{t}(a)} d \nu(a)=\max _{a \in M} W(a)
$$


Proof: Let $m=\max \{W(a): a \in M\}$. Let $\bar{a} \in M$, such that $W(\bar{a})=m$. Given $\varepsilon>0$, there exist $t_{0}$ and $\delta$, such that $W_{t}(a)>m-\varepsilon$, for any $a \in B(\bar{a}, \delta) \equiv\{a: d(a, \bar{a})<\delta\}$ and $t>t_{0}$.

Therefore, if $t>t_{0}$, we have that

$$
\int_{M} e^{t W_{t}(a)} d \nu(a) \geq \int_{B(\bar{a}, \delta)} e^{t W_{t}(a)} d \nu(a)>\nu(B(\bar{a}, \delta)) e^{t(m-\varepsilon)},
$$

thus, if $t>t_{0}$,

$$
\frac{1}{t} \log \int_{M} e^{t W_{t}(a)} d \nu(a)>\frac{1}{t} \log (\nu(B(\bar{a}, \delta)))+m-\varepsilon .
$$

Hence

$$
\liminf _{t \rightarrow+\infty} \frac{1}{t} \log \int_{M} e^{t W_{t}(a)} d \nu(a) \geq m
$$

The other inequality is analogous, using the fact that: given $\varepsilon$, there exists $t_{0}$ such that, $W_{t}(a)<m+\varepsilon$, for any $t>t_{0}$ and $a \in M$.

Proposition 10 Given a potential A Hölder continuous, we have

i)

$$
\lim _{\beta \rightarrow \infty} \frac{1}{\beta} \log \lambda_{\beta}=m(A) .
$$

ii) Any limit, in the uniform topology,

$$
V:=\lim _{n \rightarrow \infty} \frac{1}{\beta_{n}} \log \left(\psi_{\beta_{n} A}\right),
$$

is a calibrated subaction for $A$.

Proof: Let $\beta_{n}$ be a subsequence such that the following limit exists: $\frac{1}{\beta_{n}} \log \lambda_{\beta_{n}} \rightarrow k$, when $n \rightarrow \infty$. By taking a subsequence of $\beta_{n}$ we can assume that also there exists $V$ Holder, such that $V:=\lim _{n \rightarrow \infty} \frac{1}{\beta_{n}} \log \left(\psi_{\beta_{n} A}\right)$.

Given $x \in \mathcal{B}$, consider the equation

$$
\lambda_{\beta_{n}}=\frac{1}{\psi_{\beta_{n} A}(x)} \int_{M} e^{\beta_{n} A(a x)} \psi_{\beta_{n} A}(a x) d \nu(a) .
$$

It follows from lemma 1 that, when $n \rightarrow \infty$,

$$
k=\max _{a \in M}\{A(a x)+V(a x)-V(x)\} .
$$

First we show that $k \geq m(A)$ :

From the above it follows that

$$
-V(\sigma(x))+V(x)+A(x) \leq k .
$$

Let $\mu$ be a $\sigma$-invariant probability measure, then

$$
\int_{\mathcal{B}} A(x) d \mu(x)=\int_{\mathcal{B}}[-V(\sigma(x))+V(x)+A(x)] d \mu(x) \leq k .
$$

This implies $m(A) \leq k$.

Now we show that $m(A) \geq k$ :

For any $x \in \mathcal{B}$ there exist $y=a_{x} x$ such that $\sigma(y)=x$, and

$$
-V(\sigma(y))+V(y)+A(y)=k .
$$

Therefore, the compact set $K=\{y:-V(\sigma(y))+V(y)+A(y)=k\}$ is such that, $K^{\prime}=$ $\cap_{n} \sigma^{-n}(K)$ is non-empty, compact and $\sigma$-invariant. If we consider a $\sigma$-invariant probability 
measure $\mu$ with support on $K^{\prime}$, we have that $\int_{\mathcal{B}} A(y) d \mu(y)=k$. From this follows that $m(A) \geq k$.

From the above arguments $k=m(A)$ is the unique possible accumulation point of the bounded function $\beta \rightarrow \frac{1}{\beta} \log \lambda_{\beta A}$, then

$$
\lim _{\beta \rightarrow \infty} \frac{1}{\beta} \log \lambda_{\beta A}=m(A) .
$$

Moreover, from the above expressions, we can say that any limit of convergent subsequence $\lim _{n \rightarrow \infty} \frac{1}{\beta_{n}} \log \left(\psi_{\beta_{n} A}\right)$ is a calibrated subaction.

Now we return to study the Gibbs measures at zero temperature. In the case $\mu_{\beta A} \rightarrow \mu_{\infty}$, when $\beta \rightarrow \infty$ (not just a subsequence), as we said before, we have selection of probability at temperature zero (see [40], [42, 41] for general positive results and [13] [14] [22] for negative results). The next result uses the variational principle proved in the previous section and the property that the entropy of an invariant probability is not positive.

Theorem 5 Consider a Hölder potential A. Suppose that for some subsequence we have $\mu_{\beta_{n} A} \rightarrow \mu_{\infty}$. Then $\mu_{\infty}$ is a maximizing probability, i.e.,

$$
\int_{\mathcal{B}} A(x) d \mu_{\infty}(x)=m(A) .
$$

In the case the maximizing probability for A is unique, we have selection of Gibbs probability at temperature zero.

Proof. By definition, $\mu_{\beta_{n} A} \rightarrow \mu_{\infty}$, if and only if,

$$
\lim _{n \rightarrow \infty} \int_{\mathcal{B}} w d \mu_{\beta_{n} A}=\int_{\mathcal{B}} w d \mu_{\infty}, \quad \forall w \in \mathcal{C} .
$$

Now using Theorem 3 and the fact that $h(\mu) \leq 0$, we obtain

$$
\begin{aligned}
m(A) & =\lim _{\beta \rightarrow \infty} \frac{\log \lambda_{\beta A}}{\beta}=\lim _{n \rightarrow \infty}\left(\int_{\mathcal{B}} A d \mu_{\beta_{n} A}+\frac{1}{\beta_{n}} h\left(\mu_{\beta_{n} A}\right)\right) \\
& \leq \lim _{n \rightarrow \infty} \int_{\mathcal{B}} A d \mu_{\beta_{n} A}=\int_{\mathcal{B}} A d \mu_{\infty}
\end{aligned}
$$

Hence, $m(A) \leq \int_{\mathcal{B}} A d \mu_{\infty}$. Also, as $\mu_{\infty}$ is a $\sigma$-invariant measure, we have that $m(A) \geq$ $\int_{\mathcal{B}} A d \mu_{\infty}$. This implies that $m(A)=\int_{\mathcal{B}} A d \mu_{\infty}$.

Questions related to the Large Deviation property on the $X Y$ model, when $\beta \rightarrow \infty$, are considered in [42]. The existence of a calibrated subaction plays an important role in this kind of result.

We consider now a different kind of question. From an easy adaptation of Theorem 2.1 in [18] one can show:

\section{Proposition 11}

$$
\lim _{n \rightarrow \infty} \frac{1}{n} \sup _{x} \sum_{j=0}^{n-1} A\left(\sigma^{j}(x)\right)=m(A) .
$$


We consider from now on a potential $A$ which depends on two coordinates $A: S^{1} \times S^{1} \rightarrow \mathbb{R}$ of class $C^{\infty}$. A smooth real-valued function on a manifold $\mathrm{M}$ is a Morse function if it has no degenerate critical points.

In [1] it is shown that in the $C^{\infty}$ topology it is generic the set of potentials $A$ such that for any $n$ the function $\sum_{j=0}^{n-1} A \circ \sigma^{j}:\left(S^{1}\right)^{n+1} \rightarrow \mathbb{R}$ is a Morse function. In this case for each $n$ there exist a finite number of points where the values of $\frac{1}{n} \sum_{j=0}^{n-1} A \circ \sigma^{j}$ are maximal. Moreover, there exists a positive number $D$ such that for all $n$ the number of critical points of $\sum_{j=0}^{n-1} A \circ \sigma^{j}$ is smaller than $D^{n}$.

One can consider for the function $\sum_{j=0}^{n-1} A \circ \sigma^{j}:\left(S^{1}\right)^{n+1} \rightarrow \mathbb{R}$ periodic boundary conditions on $\left(S^{1}\right)^{n}$. By adapting the proof of the above result we get that the Morse property, in this case, is also true. It follows from the above proposition that, generically on the potential $A$, the maximizing probability can be approximated by probabilities with support on periodic orbits (which are isolated in $\left.\left(S^{1}\right)^{n+1}\right)$.

Other references related to the topic are [6] [8] [25]. In a future work we will analyze questions related to zeta functions for generic potentials $A$ (see [51] and [4]]).

\section{An application to the non-compact case}

An interesting example of application of the above theory is the following: consider $M_{0}=$ $\left\{z_{i}, i \in \mathbb{N}\right\}$ an increasing infinite sequence of points in $[0,1)$ and suppose that $z_{\infty}:=1=$ $\lim _{i \rightarrow \infty} z_{i}$. We will also suppose $z_{1}=0$. Therefore, each point of $M_{0}$ is isolated, and there is only one accumulating point $z_{\infty}=1$. We consider the induced euclidean metric then $M=M_{0} \cup\{1\}$ is a compact set. The state space $M_{0}$ can be identified with $\mathbb{N}$, and $M$ has a special point $z_{\infty}=1$ playing the role of the infinity. Let $\mathcal{B}_{0}=M_{0}^{\mathbb{N}}$ and $\mathcal{B}=M^{\mathbb{N}}$. Note that $\mathcal{B}_{0}$ is not compact.

Some results in Thermodynamic Formalism for the shift with countable symbols (see 55 [19) can be recovered from our previous results as we will see. We will also study the limit, when the temperature goes to zero, of Gibbs states, and some results in Ergodic Optimization will be obtained. In particular we will show the existence of sub-actions, under some suitable hypothesis. We will get this last result via limit at temperature zero of eigenfunctions at positive temperature. Results in Ergodic Optimization for this setting appear in [36], [8], [9], [38], [33], [50].

\section{Thermodynamic Formalism}

Lemma 5 Suppose that $A: \mathcal{B}_{0} \rightarrow \mathbb{R}$ is a Hölder continuous potential. Then it can be extended as a Hölder continuous function $A: \mathcal{B} \rightarrow \mathbb{R}$.

Proof: The extension is a consequence of the fact that any uniformly continuous function can be extended as a uniformly continuous function to the closure of its domain. It is easy to see that this extension is also Holder continuous.

Now let us fix an a-priori measure $\nu:=\sum_{i \in \mathbb{N}} p_{i} \delta_{z_{i}}$ on $M\left(\right.$ or $M_{0}$ ), where $p_{i}>0$ and $\sum_{i \in \mathbb{N}} p_{i}=1$. In fact, we have that $z_{\infty}=1$ belongs to the support of $\mu$, but is not an atom of $\mu$. All other points of $M$ (i.e. the points of $M_{0}$ ) are atoms for $\nu$. On this way for each Hölder continuous potential $A: \mathcal{B}_{0} \rightarrow \mathbb{R}$ we can consider the following Transfer Operator on $\mathcal{C}\left(\mathcal{B}_{0}\right)$ :

$$
\mathcal{L}_{A}(w)(x):=\int_{M} e^{A(a x)} w(a x) d \nu(a)=\sum_{i \in \mathbb{N}} e^{A\left(z_{i} x\right)} w\left(z_{i} x\right) p_{i} .
$$

Proposition 12 Let $A: \mathcal{B}_{0} \rightarrow \mathbb{R}$ be a Hölder potential. Then 
(a) there exists a positive number $\lambda_{A}$ and a positive Hölder function $\psi_{A}: \mathcal{B}_{0} \rightarrow \mathbb{R}$, such that, $\mathcal{L}_{A} \psi_{A}=\lambda_{A} \psi_{A}$.

If we consider the normalized potential $\bar{A}=A+\log \psi_{A}-\log \psi_{A} \circ \sigma-\log \lambda_{A}$, then

(b) there exists an unique fixed point $\mu_{A}$ for $\mathcal{L}_{\bar{A}}^{*}$, which is a $\sigma$-invariant probability measure on $\mathcal{B}_{0}$.

(c) the measure

$$
\rho_{A}=\frac{1}{\psi_{A}} \mu_{A}
$$

satisfies $\mathcal{L}_{A}^{*}\left(\rho_{A}\right)=\lambda_{A} \rho_{A}$. Therefore, $\rho_{A}$ is an eigen-measure for $\mathcal{L}_{A}^{*}$.

(d) for any Hölder function $w: \mathcal{B}_{0} \rightarrow \mathbb{R}$, we have that, in the uniform convergence topology,

$$
\frac{\mathcal{L}_{A}^{n}(w)}{\left(\lambda_{A}\right)^{n}} \rightarrow \psi_{A} \int_{\mathcal{B}_{0}} w d \rho_{A},
$$

and

$$
\mathcal{L}_{\bar{A}}^{n} \omega \rightarrow \int_{\mathcal{B}_{0}} \omega d \mu_{A}
$$

Proof: Using lemma 5 we can extend $\mathcal{L}_{A}$ to $\mathcal{C}(\mathcal{B})$. From Theorem 11 we obtain $\lambda_{A}>0$ and $\psi_{A}>0$ Hölder continuous such that

$$
\sum_{i} e^{A\left(z_{i} x\right)} \psi_{A}\left(z_{i} x\right) p_{i}=\lambda_{A} \cdot \psi_{A}(x) \quad \forall x \in \mathcal{B} .
$$

In particular, the restriction $\psi_{A}: \mathcal{B}_{0} \rightarrow \mathbb{R}$ will satisfy also the expression

$$
\sum_{i} e^{A\left(z_{i} x\right)} \psi_{A}\left(z_{i} x\right) p_{i}=\lambda_{A} \cdot \psi_{A}(x) \quad \forall x \in \mathcal{B}_{0} .
$$

This proves item (a).

Consider $\bar{A}=A+\log \psi_{A}-\log \psi_{A} \circ \sigma-\log \lambda_{A}$.

In order to prove item (b) we observe that from Theorem 2 there exists $\mu_{A}$ on $\mathcal{B}$ satisfying item (b). We want prove that $\mu_{A}\left(\mathcal{B}-\mathcal{B}_{0}\right)=0$, or equivalently $\mu_{A}\left(\mathcal{B}_{0}\right)=1$. On this way we only need to show that $\mu_{A}\left(\left\{x \in \mathcal{B}: x_{1}=1\right\}\right)=0$, because for all $n \geq 1$, we have

$$
\mu_{A}\left(\left\{x \in \mathcal{B}: x_{n}=1\right\}\right)=\mu_{A}\left(\sigma^{-n+1}\left(\left\{x \in \mathcal{B}: x_{1}=1\right\}\right)\right),
$$

therefore, we will have

$$
\mu_{A}\left(\mathcal{B}-\mathcal{B}_{0}\right) \leq \sum_{n=1}^{\infty} \mu_{A}\left(\left\{x \in \mathcal{B}: x_{n}=1\right\}\right)=0 .
$$

To prove that $\mu_{A}\left(\left\{x \in \mathcal{B}: x_{1}=1\right\}\right)=0$, we fix $\varepsilon>0$, and we consider a Hölder function $w_{\varepsilon}$, such that, $\chi_{\left\{x \in \mathcal{B}: x_{1}=1\right\}} \leq w_{\varepsilon} \leq 1$ and $w_{\varepsilon}(x)=0$, if $x_{1}<1-\varepsilon$. Then, using Theorem 2 item c)

$$
\begin{aligned}
& \mu_{A}\left(\left\{x \in \mathcal{B}: x_{1}=1\right\}\right) \leq \int_{\mathcal{B}} w_{\varepsilon} d \mu_{A}=\lim _{n \rightarrow+\infty} \mathcal{L}_{\bar{A}}^{n} w_{\varepsilon}\left(0^{\infty}\right) \\
& =\lim _{n \rightarrow+\infty} \sum_{i_{1}: z_{i_{1}}>1-\varepsilon} p_{i_{1}} \sum_{i_{2}, \ldots, i_{n}} e^{S_{\bar{A}}^{n}\left(z_{i_{1}} z_{i_{2}} \ldots z_{i_{n}} 0^{\infty}\right)} w_{\varepsilon}\left(z_{i_{1}} z_{i_{2}} \ldots z_{i_{n}} 0^{\infty}\right) p_{i_{2}} \ldots p_{i_{n}} \\
& \leq \lim _{n \rightarrow+\infty} \sum_{i_{1}: z_{i_{1}}>1-\varepsilon} p_{i_{1}} e^{\|\bar{A}\|} \sum_{i_{2}, \ldots, i_{n}} e^{S_{\bar{A}}^{n-1}\left(z_{i_{2}} \ldots z_{i_{n}} 0^{\infty}\right)} p_{i_{2} \ldots p_{i_{n}}}=\sum_{i_{1}: z_{i_{1}}>1-\varepsilon} p_{i_{1}} e^{\|\bar{A}\|}
\end{aligned}
$$

where we used in the last equation the normalization property.

Now the claim follows easily when we use the fact that the a-priori measure is supported on $[0,1)$, which makes

$$
\sum_{i_{1}: z_{i_{1}}>1-\varepsilon} p_{i_{1}} \rightarrow 0 \quad \text { when } \quad \varepsilon \rightarrow 0 .
$$


The items (c) and (d) follow when we restrict to $\mathcal{B}_{0}$ the result of Theorem 2 .

Now let us compare this setting with some results contained in [55]. The operator $\mathcal{L}_{A}$ can be written as

$$
\mathcal{L}_{A}(w)(x)=\sum_{i} e^{A\left(z_{i} x\right)} w\left(z_{i} x\right) p_{i}=\sum_{i} e^{A\left(z_{i} x\right)+\log \left(p_{i}\right)} w\left(z_{i} x\right)
$$

that is, the Classical Ruelle Operator with potential $B:=A+\log (P)$, where $P\left(y_{1}, y_{2}, y_{3}, \ldots\right)=$ $P\left(y_{1}\right)=p_{i}$, if, $y_{1}=z_{i}$. We denote this operator by $L_{B}$, or, $L_{A+\log (P)}$.

Clearly $(A+\log (P))\left(z_{i}, y_{2}, y_{3}, \ldots\right) \rightarrow-\infty$, when $i \rightarrow+\infty$, because $p_{i} \rightarrow 0$, when, $i \rightarrow+\infty$. Furthermore, if we define

$$
\operatorname{Var}_{n}(B)=\sup \left\{|B(x)-B(y)|: x_{1}=y_{1}, \ldots, x_{n}=y_{n}\right\},
$$

then, there exists $C>0$, such that, $\operatorname{Var}_{n}(B) \leq C \frac{1}{2^{n \alpha}}$, for any $n \geq 1$. This means that $B$ is locally Hölder continuous (see [55).

Define

$$
Z_{n}(B, a):=\sum_{\substack{\sigma^{n}(y)=y \\ y_{1}=a}} e^{S_{n} B(y)} .
$$

Proposition 13 Fix $a \in M_{0}$, then, there exists a constant $M_{a}$ and an integer $N_{a}$, such that, for any $n>N_{a}$ :

$$
\frac{Z_{n}(B, a)}{\left(\lambda_{A}\right)^{n}} \in\left[M_{a}^{-1}, M_{a}\right]
$$

Proof: Let $x=a^{\infty}=(a, a, a, a, \ldots)$. When we apply item d) of Proposition 12 for $w \equiv 1$ we get

$$
\frac{\mathcal{L}_{A}^{n}(w)(x)}{\left(\lambda_{A}\right)^{n}} \rightarrow C>0 .
$$

Remember that we denote by $a^{n}=\left(a_{n}, \ldots, a_{1}\right) \in M_{0}^{n}$, then there exist $M_{1}>0$ and $N_{a}>0$ such that for $n \geq N_{a}$ :

$$
\begin{aligned}
& \frac{1}{\left(\lambda_{A}\right)^{n+1}} \sum_{a^{n}} e^{S_{n} B\left(a^{n} x\right)} \\
& =\frac{1}{\left(\lambda_{A}\right)^{n+1}} \sum_{a^{n}} e^{S_{n} A\left(a^{n} x\right)+\log \left(P\left(a_{n}\right) \ldots P\left(a_{1}\right)\right)} \in\left[M_{1}^{-1}, M_{1}\right] .
\end{aligned}
$$

Let $y$ be the periodic point with period $n+1$ obtained by the successive concatenation of $\left(a, a_{n}, \ldots, a_{1}\right)$ and let $z=\sigma(y)$. We have

$$
\begin{aligned}
\left|S_{n} B\left(a^{n} x\right)-S_{n} B(z)\right| & =\left|S_{n} B\left(a_{n}, \ldots, a_{1}, a, a, a, \ldots\right)-S_{n} B\left(a_{n}, \ldots, a_{1}, a, a_{n}, a_{n-1}, \ldots\right)\right| \\
& =\left|S_{n} A\left(a_{n}, \ldots, a_{1}, a, a, a, \ldots\right)-S_{n} A\left(a_{n}, \ldots, a_{1}, a, a_{n}, a_{n-1}, \ldots\right)\right| \\
& \leq C\left(\frac{1}{2^{\alpha}}+\frac{1}{2^{2 \alpha}}+\ldots+\frac{1}{2^{n \alpha}}\right) \cdot d(x, y)^{\alpha} .
\end{aligned}
$$

Using the fact that $\mathcal{B}_{0}$ has finite diameter we obtain a constant $M_{2}>0$, such that,

$$
\left|S_{n} B\left(a^{n} x\right)-S_{n} B(z)\right| \leq M_{2}, \quad \forall n \in \mathbb{N}, a^{n} \in M_{0}^{n} .
$$

Furthermore, using the property $\sigma^{n}(z)=y$, we conclude that $B\left(\sigma^{n} z\right)=A\left(\sigma^{n}(z)\right)+\log (P(a))$ is bounded independently of $z$. Therefore, there exists a constant $M_{3}>0$, such that,

$$
\left|S_{n} B\left(a^{n} x\right)-S_{(n+1)} B(z)\right| \leq M_{3}, \quad \forall n \in \mathbb{N}, a^{n} \in M_{0}^{n} .
$$


Note that $S_{(n+1)} B(z)=S_{(n+1)} B(y)$. Then,

$$
\sum_{a^{n}} e^{S_{n} B\left(a^{n} x\right)-M_{3}} \leq \sum_{\substack{\sigma^{n+1}(y)=y \\ y_{1}=a}} e^{S_{(n+1)} B(y)} \leq \sum_{a^{n}} e^{S_{n} B\left(a^{n} x\right)+M_{3}}
$$

Therefore, when $n \geq N_{a}$, we get

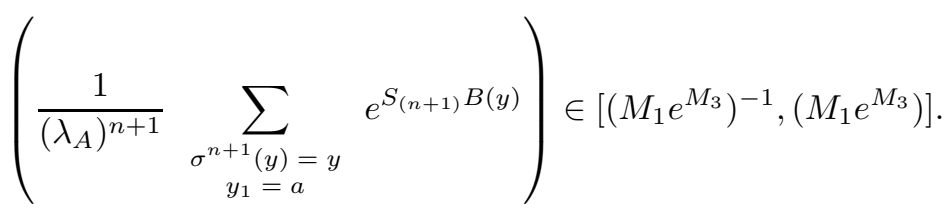

Choosing $M_{a}=M_{1} e^{M_{3}}$ we conclude the proof.

In this way, we can say that $B=A+\log (P)$ is positive recurrent (see 55 Definition 2). Following [55] Theorem 4 we get a Ruelle-Perron-Frobenius Theorem (as in Theorem 12 above). It follows from the above proposition that $\lambda_{A}$ is the Gurevic pressure of $B$ (see [55] definition 1).

We would like to point out some differences on the topology considered in our setting with the classical one used in the theory of Thermodynamic Formalism with state space $\mathbb{N}$. The set $M_{0}^{\mathbb{N}}$ can be identified with $\mathbb{N}^{\mathbb{N}}$, but the metric space $M_{0}^{\mathbb{N}}$ is different from the metric space $\mathbb{N}^{\mathbb{N}}$ with the discrete product topology. Here, we consider a distance (induced in the subset $\left.M_{0} \cup\left\{z_{\infty}\right\} \subset[0,1]\right)$, such that, for any two points $x=\left(x_{1}, x_{2}, \ldots\right), y=\left(y_{1}, y_{2}, \ldots\right) \in M_{0}^{\mathbb{N}}$

$$
d(x, y)=\sum_{n \in \mathbb{N}} \frac{1}{2^{n}} d_{[0,1]}\left(x_{n}, y_{n}\right)
$$

On the other hand, the metric considered in 55 is of the form: for two points $x, y \in \mathbb{N}^{\mathbb{N}}$

$$
\widetilde{d}(x, y)=\frac{1}{2^{n}}, \text { if } x_{1}=y_{1}, \ldots, x_{n-1}=y_{n-1}, x_{n} \neq y_{n}
$$

Using that the diameter of $[0,1]$ is one, it follows that $d(x, y) \leq \widetilde{d}(x, y)$. In particular, any convergent sequence on the metric $\widetilde{d}$ is a convergent sequence on the metric $d$, and any continuous/Hölder function $A$ for the metric $d$ is a continuous/Hölder function for the metric $\widetilde{d}$. But the same is not true in the opposite direction. This is a subtle question. Results in 55 and here are obtained under slight different hypothesis. Anyway, in physical applications this is probably a not very important point.

Considering the dual space, it follows from the relation $d(x, y) \leq \widetilde{d}(x, y)$ that any open set for the metric $d$ is an open set for the metric $\widetilde{d}$. Then, the Borel sigma-algebra generated by $d$ is contained in the Borel sigma-algebra generated by $\widetilde{d}$. on the order hand, the cylinder sets [55] are closed sets for the metric $d$, therefore, they belong to the sigma-algebra generated by $d$. In this way, the Borel sigma-algebra generated by $d$, or, by $\widetilde{d}$, is the same.

\section{Ergodic Optimization}

Once more we point out that the concepts of sub-action and maximizing measures do not involve an a-priori measure. On this way the statement of the next theorem does not use any a-priori probability. On the other hand, some condition on $A$ must be assumed in order to obtain positive results in Ergodic Optimization. For example: the potential $A: \mathcal{B}_{0} \rightarrow \mathbb{R}$, given by

$$
A(x)=-\sum_{i=1}^{\infty} \frac{1}{2^{i}} d\left(x_{i}, 1\right),
$$

does not have maximizing measures on $\mathcal{B}_{0}$. 
We need some hypothesis on $A$ in such way it prevents "the mass to go to infinity". Under some appropriate and natural conditions on $A$, we will obtain below the existence of calibrated sub-actions and maximizing measures. In the proof, we consider a limit involving a subsequence of eigenfunctions of $\mathcal{L}_{\beta A}$, when the temperature $\frac{1}{\beta}$ goes to zero.

Theorem 6 Suppose that $A: \mathcal{B}_{0} \rightarrow \mathbb{R}$ is a Hölder continuous potential. Consider the Hölder continuous extension $A: \mathcal{B} \rightarrow \mathbb{R}$. If the extension satisfies:

$$
A\left(x_{1}, \ldots, x_{n-1}, 1, x_{n+1}, x_{n+2}, \ldots\right)<A\left(x_{1}, \ldots, x_{n-1}, 0, x_{n+1}, x_{n+2}, \ldots\right)
$$

for any $n \in \mathbb{N}$ and $x_{i} \in M$, then:

a) $A$ has a calibrated subaction $V$ on $\mathcal{B}_{0}$, that means: for any $x \in \mathcal{B}_{0}$,

$$
0=\max _{a \in M_{0}}(A(a x)+V(a x)-V(x)-m(A)) .
$$

b) Any maximizing measure for $A$ has support on $\mathcal{B}_{0}$.

Proof: We define an a-priori probability measure on $M_{0}$ by the expression

$$
\nu=\sum_{i \in \mathbb{N}} p_{i} \cdot \delta_{z_{i}}
$$

where $\sum_{i \in \mathbb{N}} p_{i}=1$ and $p_{i}>0$.

Claim: Denote by $\psi_{\beta A}$ the maximal eigenfunction for the Ruelle operator given by the potential $\beta A$ and the a-priori measure $\nu$. Let $V$ be the limit of some subsequence $\frac{1}{\beta_{n}} \log \psi_{\beta_{n} A}$. Then, we have

$$
\text { (i) } \quad \psi_{\beta A}\left(1, x_{1}, x_{2}, x_{3}, \ldots\right)<\psi_{\beta A}\left(0, x_{1}, x_{2}, x_{3}, \ldots\right) \quad \forall x \in \mathcal{B}
$$

$$
\text { (ii) } \quad V\left(1, x_{1}, x_{2}, x_{3}, \ldots\right) \leq V\left(0, x_{1}, x_{2}, x_{3}, \ldots\right) \quad \forall x \in \mathcal{B}
$$

Proof of (i): We know by item (c) of the Theorem 2 that

$$
\frac{\mathcal{L}_{\beta A}^{n}(1)(x)}{\left(\lambda_{\beta A}\right)^{n}} \rightarrow \psi_{\beta A}(x) \int_{\mathcal{B}} 1 d \rho_{\beta A}, \quad \forall x \in \mathcal{B} .
$$

Hence,

$$
\rho_{\beta A}(\mathcal{B}) \psi_{\beta A}(x)=\lim _{n \rightarrow \infty} \int_{M^{n}} e^{S_{n} \beta A\left(a^{n} x\right)-n \log \lambda_{\beta A}} d \nu^{n}\left(a^{n}\right), \quad \forall x \in \mathcal{B} .
$$

Suppose now that $z=\left(1, x_{1}, x_{2}, x_{3}, \ldots\right)$ and $y=\left(0, x_{1}, x_{2}, x_{3}, \ldots\right)$, with $x \in \mathcal{B}$, then, using (16), we get

$$
\int_{M^{n}} e^{S_{n} \beta A\left(a^{n} z\right)-n \log \lambda_{\beta A}} d \nu^{n}\left(a^{n}\right)<\int_{M^{n}} e^{S_{n} \beta A\left(a^{n} y\right)-n \log \lambda_{\beta A}} d \nu^{n}\left(a^{n}\right),
$$

which implies $\psi_{\beta A}(z)<\psi_{\beta A}(y)$.

Proof of item (ii): By hypothesis $V$ satisfies

$$
V(x)=\lim _{n \rightarrow \infty} \frac{1}{\beta_{n}} \log \left(\psi_{\beta_{n} A}(x)\right),
$$

as the $\log$ function is monotone, we get $V(z) \leq V(y)$, which finishes the proof of the claim.

Now let $R_{-}=A+V-V \circ \sigma-m(A)$, where $V$ was defined above. We know, by the sub-action equation, that $R_{-} \leq 0$. From (16) and the above claim, we get that for any $x \in \mathcal{B}_{0}$ :

$$
\begin{aligned}
R_{-}(1 x) & =A(1 x)+V(1 x)-V(x)-m(A) \\
& <A(0 x)+V(0 x)-V(x)-m(A)=R_{-}(0 x) \leq 0 .
\end{aligned}
$$


Using now the fact that $V$ is a calibrated subaction $($ on $\mathcal{B})$ we conclude the proof of $(a)$, because last inequality shows that

$$
\max _{a \in M_{0}}(A(a x)+V(a x)-V(x)-m(A))=\max _{a \in M}(A(a x)+V(a x)-V(x)-m(A)) .
$$

We point out that the fact that the extension has a maximizing measure is a consequence of the compactness of $\mathcal{B}$. In order to prove (b) we will fix a $x \in \mathcal{B} \backslash \mathcal{B}_{0}$ and prove that $x$ does not belong to the support of any maximizing measure.

Note that if $R_{-}\left(\sigma^{k-1}(x)\right)<0$, then $x$ does not belong to the support of the maximizing probability $\mu$. Indeed,

$$
\int_{\mathcal{B}} R_{-} \circ \sigma^{k-1} d \mu=\int_{\mathcal{B}} R_{-} d \mu=\int_{\mathcal{B}} A d \mu-m(A)=0,
$$

which, combined to the continuity of $R_{-} \leq 0$, proves that $R_{-} \circ \sigma^{k-1}$ vanishes at the support of $\mu$. Now we will prove that $R_{-}\left(\sigma^{k-1}(x)\right)<0$. So, let $k \in \mathbb{N}$ be such that $x_{k}=1$ and $x_{l}<1$, $\forall 1 \leq l<k$. Let $y \in \mathcal{B}$ be given by $y_{i}=x_{i}$, if $i \neq k$, and $y_{k}=0$.

We have that $\sigma^{k-1}(x)=\left(1, x_{k+1}, x_{k+2}, \ldots\right)$ and $\sigma^{k-1}(y)=\left(0, x_{k+1}, x_{k+2}, \ldots\right)$, therefore

$$
\begin{aligned}
R_{-}\left(\sigma^{k-1}(x)\right) & =A\left(\sigma^{k-1}(x)\right)+V\left(\sigma^{k-1}(x)\right)-V\left(\sigma^{k}(x)\right)-m(A) \\
& <A\left(\sigma^{k-1}(y)\right)+V\left(\sigma^{k-1}(y)\right)-V\left(\sigma^{k}(y)\right)-m(A)=R_{-}\left(\sigma^{k-1}(y)\right) \leq 0,
\end{aligned}
$$

where we used above the hypothesis (16), item (ii) of the claim, and also $\sigma^{k}(x)=\sigma^{k}(y)$.

In the proof of the above theorem we show the following result:

Corollary 2 Given an a-priori probability measure $\nu=\sum_{i=1}^{\infty} p_{i} \delta_{z_{i}}, p_{i}>0$, under the hypothesis of the above theorem, then, there exists a subsequence $\left\{\beta_{n}\right\}$ and a Hölder continuous function $V: \mathcal{B}_{0} \rightarrow \mathbb{R}$, such that,

$$
\frac{1}{\beta_{n}} \log \left(\psi_{\beta_{n} A}\right) \rightarrow V
$$

uniformly on $\mathcal{B}_{0}$. Furthermore, any possible limit $V$ is a calibrated sub-action for $A$ on $\mathcal{B}_{0}$.

An example of potential satisfying the hypothesis of the above theorem is given by

$$
A(x)=-d\left(x, 0^{\infty}\right)
$$

where $0^{\infty}=(0,0,0,0 \ldots)$. Note that in the claim of hypothesis $(16)$ we can change 0 by any fixed $z_{i}$, and the result we get will be the same.

It is important to remark that when the temperature changes, then, the operator varies in a different way of what happens in the classical sense: for a fixed $\beta>0$, we have:

$$
\begin{gathered}
\int_{M} e^{\beta A(a x)} w(a x) d \nu(a)=\sum_{i} e^{\beta A\left(z_{i} x\right)} w\left(z_{i} x\right) \cdot p_{i}= \\
\sum_{i} e^{\beta A\left(z_{i} x\right)+\log \left(P\left(z_{i}\right)\right)} w\left(z_{i} x\right) .
\end{gathered}
$$

Then, (in this work) the main eigenvalue and eigenfunction, respectively, $\lambda_{\beta}>0$ and $\psi_{\beta}>0$, are associated to $\beta\left(A+\frac{\log (P)}{\beta}\right)$ (in the setting of the Classical Ruelle Operator).

In this way we can think of the function $z_{i} \rightarrow \frac{\log \left(P\left(z_{i}\right)\right)}{\beta}$ as a perturbation of the potential $A$, that goes to zero when $\beta \rightarrow \infty$ (but not uniformly). 


\section{The differentiable structure and the involution kernel}

We consider in this section the $X Y$ model. This is the case where $M=S^{1}$, and the a-priori measure is the Lebesgue measure on the circle. $\left(S^{1}\right)^{\mathbb{N}}$ has a differentiable structure.

We know that, in the case where the potential $A$ is Holder, the eigenfunction $\psi_{A}$ is also Holder and belongs to the same Holder class. The main result of this section is theorem 8, where we prove that, under mild assumptions concerning the differentiability of $A$ (see definition 6 ), the associated eigenfunction $\psi_{A}$ is differentiable in each coordinate $x_{j}$ of $x$.

In this setting, we point out that in [41] it is analyzed several questions which involve differentiability for potentials which depend just on two coordinates. Here we consider more general potentials.

Let $\mathcal{B}^{*}=\left\{\left(\ldots, y_{2}, y_{1}\right) \in\left(S^{1}\right)^{\mathbb{N}}\right\}$, and we denote by the pair

$$
(y \mid x)=\left(\ldots, y_{2}, y_{1} \mid x_{1}, x_{2} \ldots\right),
$$

the general element of $\hat{\mathcal{B}}:=\mathcal{B}^{*} \times \mathcal{B}=\left(S^{1}\right)^{\mathbb{Z}}$, the natural extension of $\mathcal{B}$. Here we will follow the ideas of [2].

We denote by $\hat{\sigma}$ the shift on $\hat{\mathcal{B}}$, i.e.

$$
\hat{\sigma}\left(\ldots, y_{2}, y_{1} \mid x_{1}, x_{2}, \ldots\right)=\left(\ldots, y_{2}, y_{1}, x_{1} \mid x_{2}, x_{3}, \ldots\right) .
$$

Definition 5 Let $A: \mathcal{B} \rightarrow \mathbb{R}$ be a continuous potential (considered as a function on $\hat{\mathcal{B}}$ ). $A$ continuous function $W: \hat{\mathcal{B}} \rightarrow \mathbb{R}$ is called an involution kernel, if

$$
A^{*}:=A \circ \hat{\sigma}^{-1}+W \circ \hat{\sigma}^{-1}-W
$$

depends only on the variable $y$.

The involution kernel is not unique.

Let us fix $x^{\prime} \in \mathcal{B}$ and $A$ a Hölder continuous potential, then we define

$$
W(y \mid x)=\sum_{n \geq 1} A\left(y_{n}, \ldots, y_{1}, x_{1}, x_{2}, \ldots\right)-A\left(y_{n}, \ldots, y_{1}, x_{1}^{\prime}, x_{2}^{\prime}, \ldots\right) .
$$

An easy calculation shows that $W(y \mid x)$ is a involution kernel (see [2]).

The Ruelle-Perron operator $\mathcal{L}_{A}$ gives two important informations: the eingenmeasure $\rho_{A}$ and the eingenfunction $\psi_{A}$. As in [2], we can use the involution Kernel in order to obtain $\psi_{A}$, if we know the eigenmeasure of the Ruelle-Perron operator associated to $A^{*}(y)$ (see proposition 14). We will show that the involution kernel allows one to differentiate $\psi_{A}$ with respect to each coordinate $x_{j}$ of $x$, using the expression of $\psi_{A}$ given in proposition 14 (see theorem 7).

Let $A: \mathcal{B} \rightarrow \mathbb{R}$ be a Hölder continuous potential and $W: \hat{\mathcal{B}} \rightarrow \mathbb{R}$ an involution kernel, then for any $a \in S^{1}, x \in \mathcal{B}$ and $y \in \mathcal{B}^{*}$, we have

$$
\left(A^{*}+W\right)(y a \mid x)=(A+W)(y \mid a x) .
$$

Questions related to Ergodic Transport Theory and the involution kernel are analyzed in 47], 17] and [43].

Remember that

$$
m(A)=\sup _{\mu \text { is } \sigma-\text { invariant }} \int_{\mathcal{B}} A d \mu
$$

and define

$$
m\left(A^{*}\right)=\sup _{\mu \text { is } \sigma^{*}-\text { invariant }} \int_{\mathcal{B}^{*}} A^{*} d \mu
$$

The next result is an adaptation to the present setting of a result in [2]. 
Lemma 6 Let $\mathcal{L}_{A}$ and $\mathcal{L}_{A^{*}}$ be the Ruelle operators defined on $\mathcal{B}$ and $\mathcal{B}^{*}$, and $W(y \mid x)$ an involution kernel.

Then, for any $x \in \mathcal{B}, y \in \mathcal{B}^{*}$, and any function $f: \hat{\mathcal{B}} \rightarrow \mathbb{R}$

$$
\mathcal{L}_{A^{*}}\left(f(\cdot \mid x) e^{W(\cdot \mid x)}\right)(y)=\mathcal{L}_{A}\left(f \circ \hat{\sigma}(y \mid \cdot) e^{W(y \mid \cdot)}\right)(x) .
$$

Proof: Under our notation we write $A(y \mid x)=A(x)$ and $A^{*}(y \mid x)=A^{*}(y)$. Consider $x \in \mathcal{B}$, $y \in \mathcal{B}^{*}$ fixed, then by the definition of $\mathcal{L}_{A}$ and $\mathcal{L}_{A^{*}}$, and equation 18 , we obtain

$$
\begin{aligned}
\mathcal{L}_{A^{*}}\left(f(\cdot \mid x) e^{W(\cdot \mid x)}\right)(y) & =\int_{S^{1}} f(y a \mid x) e^{\left(A^{*}(y a)+W(y a \mid x)\right)} d \nu(a) \\
& =\int_{S^{1}} f \circ \hat{\sigma}(y \mid a x) e^{(A(a x)+W(y \mid a x))} d \nu(a) \\
& =\mathcal{L}_{A}\left(f \circ \hat{\sigma}(y \mid \cdot) e^{W(y \mid \cdot)}\right)(x)
\end{aligned}
$$

Let $\rho_{A}$ and $\rho_{A^{*}}$ the eigenmeasures for $\mathcal{L}_{A}^{*}$ and $\mathcal{L}_{A^{*}}^{*}$, given in Theorem 2. Suppose $c$ is such that $\iint_{\mathcal{B} \times \mathcal{B}^{*}} e^{W(y \mid x)-c} d \rho_{A^{*}}(y) d \rho_{A}(x)=1$.

Proposition 14 Suppose $K(y \mid x)=e^{W(y \mid x)-c}$. Then,

$$
d \hat{\mu}_{A}=K(y \mid x) d \rho_{A^{*}}(y) d \rho_{A}(x)
$$

is invariant for $\hat{\sigma}$ and is the natural extension of the Gibbs measure $\mu_{A}$.

The function $\psi_{A}(x)=\int_{\mathcal{B}^{*}} K(y \mid x) d \rho_{A^{*}}(y)$ is the main eigenfunction for $\mathcal{L}_{A}$, and the function $\psi_{A^{*}}(y)=\int_{\mathcal{B}} K(y \mid x) d \rho_{A}(x)$ is the main eigenfunction for $\mathcal{L}_{A^{*}}$. Furthermore $\lambda_{A}=\lambda_{A^{*}}$.

Proof: We denote by $K(y \mid x)=e^{W(y \mid x)-c}$, and we define a positive function $\psi$, by the expression $\psi(x)=\int_{\mathcal{B}^{*}} K(y \mid x) d \rho_{A^{*}}(y)$. In order to prove that $\psi$ is an eigenfunction for $\mathcal{L}_{A}$, we remember that $\mathcal{L}_{A^{*}}^{*}\left(\rho_{A^{*}}\right)=\lambda_{A^{*}} \rho_{A^{*}}$, hence

$$
\begin{aligned}
\psi(x) & =\int_{\mathcal{B}^{*}} K(y \mid x) d\left(\frac{1}{\lambda_{A^{*}}} \mathcal{L}_{A}^{*}\left(\rho_{A^{*}}\right)\right)(y)=\int_{\mathcal{B}^{*}} \frac{1}{\lambda_{A^{*}}} \mathcal{L}_{A^{*}}(K(\cdot \mid x))(y) d \rho_{A^{*}}(y) \\
& =\int_{\mathcal{B}^{*}} \frac{1}{\lambda_{A^{*}}} \mathcal{L}_{A}(K(y \mid \cdot))(x) d \rho_{A^{*}}(y)=\frac{1}{\lambda_{A^{*}}} \mathcal{L}_{A}(\psi)(x),
\end{aligned}
$$

where in the third equality we have used equation (19) with $f=1$. This means that $\psi$ is a positive eigenfunction for $\mathcal{L}_{A}$, now using Proposition 1 we get that $\psi=\psi_{A}$ and $\lambda_{A^{*}}=\lambda_{A}$. The proof for the case of $\psi_{A}^{*}$ is similar.

By the same arguments used above, for any bounded Borel $f: \mathcal{B}^{*} \times \mathcal{B} \rightarrow \mathbb{R}$, we have

$$
\begin{aligned}
& \int_{\mathcal{B}} \int_{\mathcal{B}^{*}} f \circ \hat{\sigma}(y \mid x) K(y \mid x) d \rho_{A^{*}}(y) d \rho_{A}(x) \\
& =\int_{\mathcal{B}^{*}} d \rho_{A^{*}}(y) \int_{\mathcal{B}} \frac{1}{\lambda_{A}} \mathcal{L}_{A}(f \circ \hat{\sigma}(y \mid \cdot) K(y \mid \cdot))(x) d \rho_{A}(x) \\
& =\int_{\mathcal{B}} d \rho_{A}(x) \int_{\mathcal{B}^{*}} \frac{1}{\lambda_{A^{*}}} \mathcal{L}_{A^{*}}(f(\cdot \mid x) K(\cdot \mid x)) d \rho_{A^{*}}(y) \\
& =\int_{\mathcal{B}} \int_{\mathcal{B}^{*}} f(y \mid x) K(y \mid x) d \rho_{A^{*}}(y) d \rho_{A}(x),
\end{aligned}
$$

hence $d \hat{\mu}_{A}=K(y \mid x) d \rho_{A^{*}}(y) d \rho_{A}(x)$ is invariant for $\hat{\sigma}$.

Finally, let us prove that $\hat{\mu}_{A}$ is the natural extension of $\mu_{A}$. Given a function $f(x)$ we get that

$$
\begin{aligned}
\int_{\mathcal{B}} \int_{\mathcal{B}^{*}} f(x) d \hat{\mu}_{A}(y, x) & =\int_{\mathcal{B}} \int_{\mathcal{B}^{*}} f(x) K(y \mid x) d \rho_{A^{*}}(y) d \rho_{A}(x) \\
& =\int_{\mathcal{B}} f(x) \psi_{A}(x) d \rho_{A}(x)=\int_{\mathcal{B}} f(x) d \mu_{A}(x) .
\end{aligned}
$$


Therefore, the measure $d \hat{\mu}_{A}(y, x)=K(y \mid x) d \rho_{A^{*}}(y) d \rho_{A}(x)$ projects onto $\mu_{A}$ and $\mu_{A^{*}}$ (by the same arguments). The probability $\hat{\mu}_{A}$ is therefore the natural extension of $\mu_{A}$.

Remark: Note that, as $\lambda_{\beta A}=\lambda_{\beta A^{*}}$, we have that $m(A)=\lim _{\beta \rightarrow \infty} \frac{1}{\beta} \log \lambda_{\beta A}=m\left(A^{*}\right)$.

Definition 6 Suppose that $A$ is Lipschitz continuous and satisfies the both conditions described below:

(a) $A$ is differentiable in each coordinate $x_{j}$ of $x \in \mathcal{B}$,

(b) given $\varepsilon>0$, there exists $H_{\varepsilon}>0$, such that, for all $x$, if $|h|<H_{\varepsilon}$, then

$$
\left|\frac{A\left(x+h e_{j}\right)-A(x)}{h}-D_{j} A(x)\right| \leq \frac{\varepsilon}{2^{j}} \forall j \in \mathbb{N},
$$

where $D_{j} A(x)$ denote the derivative of $A$ with respect to the $j$-th coordinate.

We will denote the class of such potentials by $\mathcal{D}$.

The potential $A(x)=\sum_{n=1}^{\infty} \frac{1}{2^{n}} \sin \left(x_{n}+1 / 2^{n}\right)$ belongs to the class $\mathcal{D}$. Moreover, any potential which depends on finite coordinates and is of class $C^{2}$ belongs to $\mathcal{D}$.

Proposition 15 Suppose that A belongs to the class $\mathcal{D}$. Given an involution kernel $W$ we have that for any $j$

$$
\frac{\partial}{\partial x_{j}} W(y \mid x)=\sum_{n \geq 1} D_{n+j} A\left(y_{n}, \ldots, y_{1}, x_{1}, x_{2}, \ldots\right) .
$$

Proof: Let us first prove that the sum in the right hand side is convergent. Indeed, using that $A$ is Lipschitz, there exist $K>0$ such that

$$
|A(x)-A(\tilde{x})|<K d(x, \tilde{x}) .
$$

If we denote $x_{h}^{j}=x+h e_{j}$ and $y^{n} x=\left(y_{n}, \ldots, y_{1}, x_{1}, x_{2}, \ldots\right)$, then we have

$$
\left|\frac{A\left(y^{n} x+h e_{j+n}\right)-A\left(y^{n} x\right)}{h}\right| \leq \frac{K}{h} \frac{d_{S^{1}}\left(x_{j}+h, x_{j}\right)}{2^{n+j}}=\frac{K}{2^{n+j}} .
$$

Using that $A$ belongs to $\mathcal{D}$, we get that, given $\varepsilon>0$, there exists $H_{\varepsilon}>0$ such that, for each $|h|<H_{\varepsilon}$, we have

$$
\left|D_{n+j} A\left(y^{n} x\right)\right| \leq\left|\frac{A\left(y^{n} x_{h}^{j}\right)-A\left(y^{n} x\right)}{h}\right|+\frac{\varepsilon}{2^{n+j}} \leq \frac{K}{2^{n+j}}+\frac{\varepsilon}{2^{n+j}},
$$

which implies

$$
\sum_{n \geq 1}\left|D_{n+j} A\left(y^{n} x\right)\right|<\frac{K+\varepsilon}{2^{j}}<\infty .
$$

Now, we will prove the proposition.

$$
\begin{aligned}
& \left|\frac{W\left(y \mid\left(x+h e_{j}\right)\right)-W(y \mid x)}{h}-\sum_{n \geq 1} D_{n+j} A\left(y^{n} x\right)\right| \\
& =\left|\frac{1}{h}\left(\sum_{n \geq 1} A\left(y^{n} x+h e_{j+n}\right)-A\left(y^{n} x\right)\right)-\sum_{n \geq 1} D_{n+j} A\left(y^{n} x\right)\right| \\
& =\left|\sum_{n \geq 1}\left(\frac{A\left(y^{n} x+h e_{j+n}\right)-A\left(y^{n} x\right)}{h}-D_{n+j} A\left(y^{n} x\right)\right)\right| \leq \sum_{n \geq 1} \frac{\varepsilon}{2^{n+j}}=\frac{\varepsilon}{2^{j}},
\end{aligned}
$$


for all $|h| \leq H_{\varepsilon}$, as $A$ belongs to $\mathcal{D}$.

From the final part of the last proof we have that, for all $|h| \leq H_{\varepsilon}$, and for all $x$ and $y$,

$$
\left|\frac{W\left(y \mid\left(x+h e_{j}\right)\right)-W(y \mid x)}{h}-\frac{\partial W(y \mid x)}{\partial x_{j}}\right| \leq \frac{\varepsilon}{2^{j}} .
$$

Theorem 7 Let $\psi_{A}(x)=\int_{\mathcal{B}^{*}} e^{W(y \mid x)-c} d \rho_{A^{*}}(y)$, and suppose $A$ belongs to the class $\mathcal{D}$. Then, the eigenfunction $\psi_{A}$ is differentiable in each coordinate $x_{j}$. Moreover,

$$
\frac{\partial}{\partial x_{j}} \psi_{A}(x)=\int_{\mathcal{B}^{*}} e^{W(y \mid x)-c} \sum_{n \geq 1} D_{n+j} A\left(y_{n}, \ldots, y_{1}, x_{1}, x_{2}, \ldots\right) d \rho_{A^{*}}(y) .
$$

Proof: Consider $j \in \mathbb{N}$. We have

$$
\begin{aligned}
& \left|\frac{\psi_{A}\left(x+h e_{j}\right)-\psi_{A}(x)}{h}-\int_{\mathcal{B}^{*}} e^{W(y \mid x)-c} \frac{\partial W(y \mid x)}{\partial x_{j}} d \rho_{A^{*}}(y)\right| \\
& =\left|\int_{\mathcal{B}^{*}}\left(\frac{e^{W\left(y \mid x+h e_{j}\right)-c}-e^{W(y \mid x)-c}}{h}-e^{W(y \mid x)-c} \frac{\partial W(y \mid x)}{\partial x_{j}}\right) d \rho_{A^{*}}(y)\right| \\
& =\left|\int_{\mathcal{B}^{*}} e^{W(y \mid x)-c}\left(\frac{e^{W\left(y \mid x+h e_{j}\right)-W(y \mid x)}-1}{h}-\frac{\partial W(y \mid x)}{\partial x_{j}}\right) d \rho_{A^{*}}(y)\right| \\
& \leq\left|\int_{\mathcal{B}^{*}} e^{W(y \mid x)-c}\left(\frac{e^{W\left(y \mid x+h e_{j}\right)-W(y \mid x)}-1}{h}-\frac{W\left(y \mid x+h e_{j}\right)-W(y \mid x)}{h}\right) d \rho_{A^{*}}(y)\right| \\
& +\left|\int_{\mathcal{B}^{*}} e^{W(y \mid x)-c}\left(\frac{W\left(y \mid x+h e_{j}\right)-W(y \mid x)}{h}-\frac{\partial W(y \mid x)}{\partial x_{j}}\right) d \rho_{A^{*}}(y)\right|
\end{aligned}
$$

Now, observe that the second integral above goes to zero when $h \rightarrow 0$, as a consequence of equation (21). The first integral also goes to zero when $h \rightarrow 0$ because, using the fact that

$$
\frac{e^{a h}-1}{h}-a=\sum_{k \geq 2} \frac{a^{k} h^{k-1}}{k !},
$$

with $a=\frac{W\left(y \mid x+h e_{j}\right)-W(y \mid x)}{h}$, we have

$$
\begin{aligned}
& \left|\frac{e^{W\left(y \mid x+h e_{j}\right)-W(y \mid x)}-1}{h}-\frac{W\left(y \mid x+h e_{j}\right)-W(y \mid x)}{h}\right|=\left|\sum_{k \geq 2} \frac{\left(W\left(y \mid x+h e_{j}\right)-W(y \mid x)\right)^{k}}{k ! h}\right| \\
& =\left|\sum_{k \geq 2} \frac{\left(W\left(y \mid x+h e_{j}\right)-W(y \mid x)\right)^{k-1}}{k !} \frac{\left(W\left(y \mid x+h e_{j}\right)-W(y \mid x)\right)}{h}\right| \rightarrow 0 \text { when } h \rightarrow 0 .
\end{aligned}
$$

In the last expression we used the uniform continuity of $W$ and also (21).

Remark: In the case where $A$ depends only on the two first coordinates, we have that (see (13))

$$
\psi_{A}\left(x_{1}\right)=\frac{1}{\lambda_{A}} \int_{S^{1}} e^{A\left(y_{1}, x_{1}\right)} \psi_{A}\left(y_{1}\right) d \nu\left(y_{1}\right) .
$$

Hence, $\psi_{A}$ satisfies the equation

$$
\frac{\partial}{\partial x_{1}} \psi_{A}\left(x_{1}\right)=\frac{1}{\lambda_{A}} \int_{S^{1}} e^{A\left(y_{1}, x_{1}\right)} D_{2} A\left(y_{1}, x_{1}\right) \psi_{A}\left(y_{1}\right) d \nu\left(y_{1}\right) .
$$




\section{References}

[1] M. Asaoka, T. Fukaya, K. Mitsui and M. Tsukamoto, Growth of critical points in onedimensional lattice systems, preprint Arxiv 2012.

[2] A. Baraviera, A. O. Lopes and P. Thieullen, A large deviation principle for equilibrium states of Hölder potencials: the zero temperature case, Stochastics and Dynamics 6 (2006), $77-96$.

[3] A. T. Baraviera, L. Cioletti, A. O. Lopes, J. Mohr and R. R. Souza, On the general one-dimensional XY model: positive and zero temperature, selection and non-selection. Rev.Math. Phis. 23 (2011),no. 10, 1063-1113, 82Bxx.

[4] A. T. Baraviera, A. O. Lopes and J. K. Mengue, On the selection of subaction and measure for a subclass of potentials defined by P. Walters. Ergodic Theory and Dynamical Systems, Available on CJO2012.

[5] P. Bernard and G. Contreras. A Generic Property of Families of Lagrangian Systems. Annals of Math. Vol. 167, No. 3, 2008.

[6] M. Bertelson, Topological invariant for discrete group actions, Lett. Math. Phys. 62 (2004), $147-156$

[7] M. Bertelson and M. Gromov, Dynamical Morse entropy, Modern dynamical systems and applications, 27-44, Cambridge Univ. Press, Cambridge, 2004.

[8] R. Bissacot and E. Garibaldi, Weak KAM methods and ergodic optimal problems for countable Markov shifts, Bull. Braz. Math. Soc. 41, N 3, 321-338, 210.

[9] R. Bissacot and R. Freire Jr., On the existence of maximizing measures for irreducible countable Markov shifts: a dynamical proof, to appear in Ergodic Theory and Dynamical Systems

[10] T. Bousch, La condition de Walters, Ann. Sci. École Norm. Sup. (4) 34 (2001), no. 2, $287-311$.

[11] W. Chou and R. J. Duffin, An additive eigenvalue problem of physics related to linear programming, Advances in Applied Mathematics 8, 486-498, 1987.

[12] W. Chou and R. Griffiths, Ground states of one-dimensional systems using effective potentials, Physical Review B, Vol. 34, N 9, 6219-6234, 1986

[13] J.R. Chazottes, J.M. Gambaudo and E. Ulgade, Zero-temperature limit of one dimensional Gibbs states via renormalization: the case of locally constant potentials, Erg. Theo. and Dyn. Sys. 31 (2011), no. 4, 1109-1161

[14] J. R. Chazottes and M. Hochman, On the zero-temperature limit of Gibbs states, Commun. Math. Phys. vol 297, N1, 2010.

[15] G. Contreras and R. Iturriaga. Global minimizers of autonomous Lagrangians, $22^{\circ}$ Colóquio Brasileiro de Matemática, IMPA, 1999.

[16] G. Contreras, A. O. Lopes and Ph. Thieullen. Lyapunov minimizing measures for expanding maps of the circle, Ergodic Theory and Dynamical Systems, Vol 21, 1379-1409, 2001.

[17] G. Contreras, A. Lopes and E. Oliveira, Ergodic Transport Theory, periodic maximizing probabilities and the twist condition, to appear in "Modeling, Optimization, Dynamics and Bioeconomy", Springer Proceedings in Mathematics, Edit. David Zilberman and Alberto Pinto. 
[18] J. P. Conze and Y. Guivarc'h, Croissance des sommes ergodiques et principe variationnel, manuscript circa (1993).

[19] Y. Daon, Bernoullicity of equilibrium measures on countable Markov shifts, preprint Arxiv 2012.

[20] A. C. D. van Enter, R. Fernandez and A. D. Sokal, Regularity properties and pathologies of position-space renormalization-group transformations: Scope and limitations of Gibbsian theory, Journ. of Stat. Phys. V.72, N 5/6 879-1187, 1993.

[21] R. Ellis, Entropy, Large Deviations, and Statistical Mechanics, Springer Verlag, 2005

[22] A. C. D. van Enter and W. M. Ruszel, Chaotic Temperature Dependence at Zero Temperature, Journal of Statistical Physics, Vol. 127, No. 3, 567-573, 2007.

[23] A. Fathi, Théorème KAM faible et théorie de Mather sur les systèmes lagrangiens, Comptes Rendus de l'Académie des Sciences, Série I, Mathématique Vol 324 1043-1046, 1997.

[24] Y. Fukui and M. Horiguchi, One-dimensional Chiral $X Y$ Model at finite temperature, Interdisciplinary Information Sciences, Vol 1, 133-149, N. 2 (1995)

[25] T. Fukaya and M. Tsukamoto, Asymptotic distribution of critical values. Geom. Dedicata 143 (2009), 63-67

[26] G. Gallavotti, Statistical Mechanics: A Short Treatise, Springer Verlag, (2010)

[27] E. Garibaldi and A. O. Lopes, On Aubry-Mather theory for symbolic Dynamics, Ergodic Theory and Dynamical Systems, Vol 28 , Issue 3, 791-815 (2008)

[28] E. Garibaldi and A. O. Lopes, The effective potential and transshipment in thermodynamic formalism at temperature zero, Vol 13 - N 1, 1250009 (13 pages) Stoch. and Dyn. (2013).

[29] H.-O. Georgii, Gibbs Measures and Phase Transitions. de Gruyter, Berlin, (1988).

[30] D. A. Gomes, A. O. Lopes and J. Mohr, The Mather measure and a large deviation principle for the entropy penalized method. Commun. Contemp. Math. 13 (2011), no.2, $235-268$

[31] D. A. Gomes and E. Valdinoci, Entropy Penalization Methods for Hamilton-Jacobi Equations, Adv. Math. (2007) 215, No. 1, 94-152.

[32] M. Gromov, Singularities, expanders and topology of maps. Part 2: From combinatorics to topology via algebraic isoperimetry. Geom. Funct. Anal. 20 (2010), no. 2, 416-526.

[33] G. Iommi, Ergodic Optimization for Renewal Type Shifts, Monatshefte für Mathematik, Volume 150, Number 2 (2007), 91-95,

[34] R. B. Israel, Convexity in the theory of lattice gases, Princeton University Press, 1979.

[35] O. Jenkinson. Ergodic optimization, Discrete and Continuous Dynamical Systems, Series A, V. 15, 197-224, 2006.

[36] O. Jenkinson, R. D. Mauldin and M. Urbanski, Zero Temperature Limits of GibbsEquilibrium States for Countable Alphabet Subshifts of Finite Type, Journ. of Statis. Physics, Volume 119, Numbers 3-4 (2005),

[37] G. Keller, Gibbs States in Ergodic Theory, Cambrige Press, 1998.

[38] T. Kempton, Zero Temperature Limits of Gibbs Equilibrium States for Countable Markov Shifts, J. Stat Phys 143, 795-806, 2011. 
[39] O. Lanford, Entropy and Equilibrium States in Classical Statistical Mechanics. Statistical mechanics and mathematical problems. Battelle Rencontres, Seattle, Wash., 1971. Lecture Notes in Physics, 20. Springer-Verlag, Berlin-New York, 1973.

[40] R. Leplaideur, A dynamical proof for convergence of Gibbs measures at temperature zero, Nonlinearity 18, N 6, 2847-2880, 2005.

[41] A. O. Lopes, J. Mohr, R. Souza and Ph. Thieullen, Negative entropy, zero temperature and stationary Markov chains on the interval, Bulletin of the Brazilian Mathematical Society 40, 1-52, 2009.

[42] A. Lopes and J. Mengue, Selection of measure and a Large Deviation Principle for the general one-dimensional XY model, to appear in Dyn. Syst.

[43] A. Lopes and J. Mengue, Duality Theorems in Ergodic Transport, Journal of Statistical Physics. Vol 149, issue 5, pp 921-942 (2012)

[44] A. O. Lopes and J. Mengue, Zeta measures and Thermodynamic Formalism for temperature zero, Bulletin of the Brazilian Mathematical Society 41 (3) pp 449-480 (2010)

[45] A. O. Lopes and E. Oliveira, Entropy and variational principles for holonomic probabilities of IFS, Disc. and Cont. Dyn. Systems series A, vol 23, N 3, 937-955, 2009.

[46] A. O. Lopes, An analogy of charge distribution on Julia sets with the Brownian motion, J. Math. Phys. 30 9, 2120-2124, 1989.

[47] A. O. Lopes, E. R. Oliveira and Ph. Thieullen, The dual potential, the involution kernel and transport in ergodic optimization, preprint, 2008.

[48] R. Mañé, The Hausdorff dimension of invariant probabilities of rational maps. Lecture Notes in Math. vol.1331, 86-117, 1988.

[49] D. H. Mayer, The Ruelle-Araki transfer operator in classical statistical mechanics, LNP 123, Springer Verlag 1980

[50] I. D. Morris, Entropy for Zero-Temperature Limits of Gibbs-Equilibrium States for Countable-Alphabet Subshifts of Finite Type, Journ. of Statis. Physics, Volume 126, Number 2 (2007), 315-324,

[51] W. Parry and M. Pollicott. Zeta functions and the periodic orbit structure of hyperbolic dynamics, Astérisque Vol 187-188 1990

[52] M. Pollicott and M. Yuri, Dynamical systems and Ergodic Theory, Cambrige Press, 1998

[53] D. Ruelle, Thermodynamic Formalism, second edition, Cambridge, 2004.

[54] B. Simon, The Statistical Mechanics of Lattice Gases, Princeton Univ Press, 1993

[55] O. Sarig, Thermodynamic formalism for countable Markov shifts, Ergodic Theory and Dynamical Systems 19, 1565-1593, 1999

[56] O. Sarig, Lecture notes on thermodynamic formalism for topological Markov shifts, Penn State, 2009.

[57] R. R. Souza, Sub-actions for weakly hyperbolic one-dimensional systems, Dynamical Systems 18 (2), 165-179, 2003.

[58] C. Thompson, Infinite-Spin Ising Model in one dimension. Journal of Mathematical Physics. (9): N.2 241-245, 1968. 